\title{
Assessing the ichthyofaunal diversity and trophic level from trawl bycatch of Chennai Fishing Harbour, Southeast Coast of India
}

\author{
Paramasivam Kodeeswaran ${ }^{\mathrm{a}, *}$, Natarajan Jayakumar ${ }^{\mathrm{a}}$, Lakshmanan Ranjith ${ }^{\mathrm{b}}$ \\ a Department of Fisheries Resource Management, Dr. M.G.R. Fisheries College and Research Institute, Ponneri - 601204, Tamil Nadu, India \\ ${ }^{\mathrm{b}}$ Marine Biodiversity Division, Tuticorin Regional Station, ICAR - Central Marine Fisheries Research Institute, Thoothukudi - 628 001, Tamil \\ Nadu, India
}

\section{A R T I C L E I N F O}

\section{Article history:}

Received 14 May 2020

Received in revised form 16 October 2020

Accepted 3 November 2020

Available online 5 November 2020

\section{Keywords:}

Bottom trawling

Bycatch

Biodiversity indices

Trophic level

\begin{abstract}
A B S T R A C T
The present study aimed to document the temporal diversity and trophic level of ichthyofauna from the Chennai Fishing Harbour, Southeast coast of India. The trawl bycatch consists of 45,527 ichthyofauna individuals collected fortnightly during the period June 2018 to April 2019. The recorded ichthyofaunal diversity includes 156 species belonging to 2 classes, 14 orders, 66 families, and 119 genera. The order Perciformes dominantes with 74 species (47\%) followed by Scorpaeniformes (12\%; 19 species) and Tetraodontiformes (11\%; 18 species). Temporal diversity analysis revealed that the maximum species diversity was observed during the North-east monsoon (123 species) while the minimum was observed during the post-monsoon (107 species) period. Numerically dominant bycatch species were found to be Leiognathus equulus (6\%), Equulites lineolatus (5\%), Gazza achlamys (5\%), Karalla dussumieri, (4\%) Otolithes ruber (3\%) and Nibea maculata (2\%). Biodiversity analysis revealed that the Shannon-Wiener species diversity index ranged from 5.37 (South-west monsoon) to 5.53 (North-east monsoon) whereas the species evenness was observed from 0.77 (South-west monsoon) to 0.81 (Post-monsoon). The habitatwise diversity analysis revealed that demersal species group was dominant (72 species; $46 \%$ ) followed by reef-associated (70 species; $45 \%$ ), benthopelagic ( 8 species; $5 \%$ ), pelagic ( 5 species; $3 \%$ ) and bathydemersal (1 species; $1 \%)$. The results of the trophic level shows that the bycatch fish species was dominated by top-level carnivores (49\%) followed by mid-level carnivores (26\%), apex predator (19\%), primary carnivores (5\%) and herbivores or planktivores (1\%). The IUCN categories of bycatch species are mostly classified as Not Evaluated, Least Concern, Data Deficient and Vulnerable viz., 58\%, 36\%, 5\% and $1 \%$ respectively. The present study also gives information on juvenile fish that are encountered in trawl bycatch along the Chennai coast. The outcome of the study gives baseline information on the bycatch diversity which paves the way for the sustainable exploitation and also the management of exploited fisheries resources in this region.
\end{abstract}

(c) 2020 Elsevier B.V. All rights reserved.

\section{Introduction}

The global fish production accounted for 178.5 million tonnes during 2018 of which $87.6 \%$ provided beneficial nutrition to human beings and the remaining $12.4 \%$ was accounted for production of non-food products such as fish meals and fish oils (FAO, 2020). Meanwhile, global marine fish bycatch was estimated to be 9.1 million tonnes in the year 2014 of which bottom trawlers alone contributed 46\% (4.2 million tonnes) which constituted $10.8 \%$ of the total marine fish production (Perez Roda, 2019). In most of the maritime states of India, high portions of juveniles of commercially important fishes were landed as bycatch from bottom trawlers (Luther and Appanasastry, 1993).

\footnotetext{
* Corresponding author.

E-mail address: kodyvenkat1995@gmail.com (P. Kodeeswaran).
}

Sivasubramaniam (1990) revealed that more than $50 \%$ of bycatch comprised immature fishes, which showed the gradual decline in long lifespan species like snappers, groupers and croakers. The estimates from shrimp trawlers of the maritime states of Indian coast, revealed that bycatch constituted $40 \%$ of juveniles of commercially important organisms (Pillai, 1998). Salagrama (1998) revealed that the bycatch is retained and returned back as low value fish which are utilized as poultry feed.

Bottom trawling is a pervasive source for benthic habitat, diversity, trophic structure, and community disturbance in the continental shelf of seas (Kaiser et al., 2002; Eastwood et al., 2007; Bijukumar and Deepthi, 2009; Foden et al., 2011; Ranjith et al., 2018; Ramkumar et al., 2019). Benthic habitat not only provides shelter and refuges for the juvenile but also serves as a major food source for demersal fishes (Kaiser et al., 2002). The recurrent disturbance to the benthic habitats leads to disruption in the trophic structure of the target and non-target fish, which 


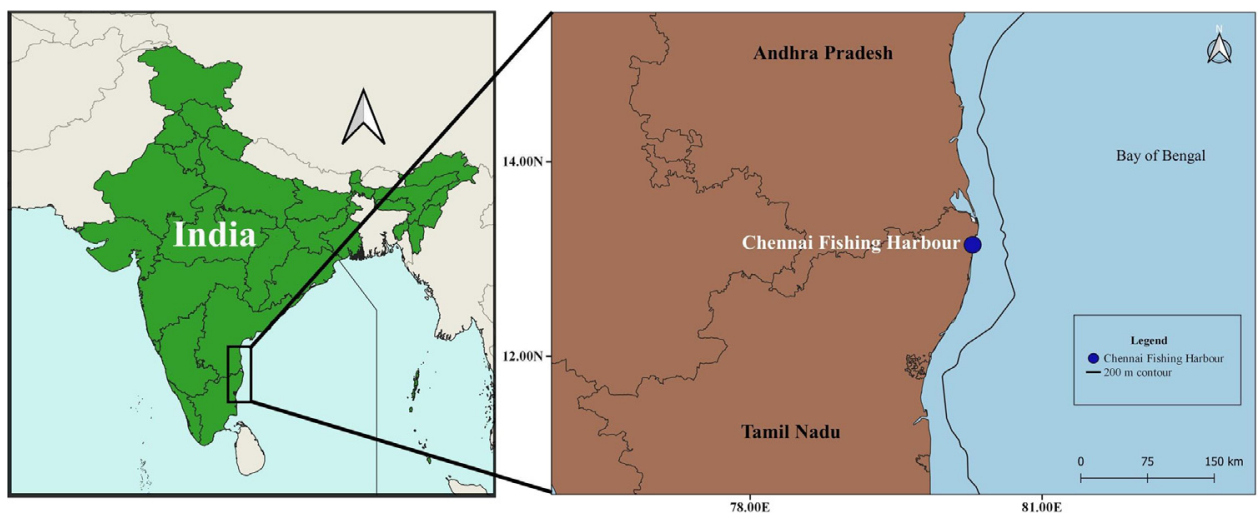

Fig. 1. Map showing the sample collection area (blue dot) in Chennai, Southeast coast of India.

effects dwindle in marine fish production (Van Denderen, 2015). In India, George et al. (1981) initially estimated the quantity of bycatches exploited by shrimp trawlers, as 315,902 tonnes (79.18\%) of total landings epitomized as bycatch with Gujarat contributing a maximum with $92.58 \%$, followed by Tamil Nadu (91.04\%) and Pondicherry (86.52\%). Sukumaran et al. (1982) reported that $85 \%$ of catch of shrimp trawlers consisted of bycatch along Mangalore and Malpe during 1980 to 82. Menon (1996) estimated that an annual bycatch landing of 43,000 tonnes along the south Indian coastal states like Tamil Nadu, Kerala, and Karnataka. The target and bycatch ratio was estimated to be 1:4.6 and 1:1.26 for the southeast coast of India during 1999 (Menon et al., 2000). The studies on the trawl bycatch from Indian waters were done by various researchers (Karnataka coast, George et al., 1981; Sukumaran et al., 1982; Southern coasts, Menon, 1996; Menon et al., 2000; Kerala coast, Kurup et al., 2003; Bijukumar and Deepthi, 2009; Dineshbabu et al., 2013; North Tamil Nadu coast, Pillai et al., 2014; Cuddalore and Parangipettai coast, Murugesan et al., 2013; Sambandamoorthy et al., 2015; Goa coast, Velip and Rivonker, 2015; Mumbai coast, Samanta et al., 2018; Thoothukudi coast, Ranjith et al., 2018; Maharashtra coast, Ramkumar et al., 2019; Karnataka coast, Mahesh et al., 2019). Nevertheless, there were few or scanty reports (Murugesan et al., 2013; Pillai et al., 2014; Sambandamoorthy et al., 2015); on the quantity of trawl bycatch from the Coromandel Coast more specifically along the Chennai coast. Keeping all the above facts in mind, the present study was designed with the objective of providing detailed reports on bycatch fishery along the Chennai coast. It also aims to identify and quantify the ichthyofaunal diversity, the trophic level and its temporal variation of trawl bycatches along the Chennai coast, Southeast coast of India.

\section{Materials and methods}

\subsection{Description of study area}

Chennai Fishing Harbour ( $13^{\circ} 07^{\prime} 24.49^{\prime \prime} \mathrm{N}$; $\left.80^{\circ} 17^{\prime} 52.20^{\prime \prime} \mathrm{E}\right)$ is located at Chennai, Southeast coast of India (Fig. 1). It is one of the major fishing harbours along the east coast of India and more than 1000 trawlers are operated for single day and multi-day fishing. The fishing ground is located at a depth of 10 to $70 \mathrm{~m}$ at the distance of 15 to $80 \mathrm{~km}$ from the shoreline, which stretches from Nagapattinam, Tamil Nadu to Ongole, Andhra Pradesh.

\subsection{Sampling methodology}

The bycatch data on finfish species were collected randomly from the trawlers of the Chennai Fishing Harbour on a fortnightly interval during the period June 2018 to April 2019 from the commercial fishing boats operating bottom trawl nets with a codend mesh size of $35 \mathrm{~mm}$. The crew members of the trawlers were requested to retain the unsorted condition of bycatch in the last haul of the day. The samples were collected from the unsorted portion and segregated into different groups viz., finfishes, crustaceans, gastropods, echinoderms, and marine debris. In case, the bycatch quantity was more, the last haul was further sub-sampled for the collection of bycatch data and raised to the total bycatch wherein sampling of large-sized fishes were excluded (Stobutzki et al., 2001). The samples collected from the observed number of boats ( $n=4$ to 6 ) during the sampling day was stretched to whole boats landed on a particular day and raised to month based on the number of fishing days in a month (Sekharan, 1962; Ramkumar et al., 2015). In this the total sampling effort consisting of one twenty one $(\mathrm{n}=121)$ on a fortnightly mode encompasses 8 to 12 hauls in a month. The samples were not collected during the first half of June 2018 and second half of April, 2019 due to an existing fishing ban that was imposed by the Government of India during the period April 15 to June 15 along the east coast of India.

\subsection{Identification of bycatch fish species}

The collected bycatch fish specimens were brought to the laboratory in ice boxes and kept deep-frozen for identification. Prior to identification, each sample was sorted, counted, weighed, and photographed fresh and later, the morphological characters of the fish specimens were examined. Bycatch fish species were identified to species level using classical taxonomic methods viz., morphology, meristic, morphometric, colours, patterns as described by various researchers in the field of ichthyology (Day, 1878; Fischer and Whitehead, 1974; Fischer and Bianchi, 1984; Talwar and Kacker, 1984; Rema Devi, 1992; Gon and Randall, 2003). The species names were validated by referring to the World Register of Marine Species (Horton et al., 2018) and FishBase (Froese and Pauly, 2018). The total length of all the fish specimens was measured using a digital caliper (Mitutoyo CD6"ASX) to the nearest $0.1 \mathrm{~mm}$. Fin clips were preserved in $99 \%$ ethanol for new records and rare species for long-term preservation and barcoding studies. The new distributional records and rare fish specimens were preserved in $10 \%$ formalin and deposited in the Fisheries Museum of Dr. M.G.R Fisheries College and Research Institute, Ponneri, Tamil Nadu (Kodeeswaran et al., 2020a,c).

\subsection{Biodiversity assessment}

The abundance data collected were classified into four seasons viz., Summer (April, May, June); South-west monsoon (July, August, September); North-east monsoon (October, November, 
December) and Post-monsoon (January, February, March) for the biodiversity assessment studies (Pillai et al., 2014). From the seasonal species abundance data, the biodiversity indices were calculated using PRIMER v7 (Plymouth Routines In Multivariate Ecological Research) software package developed at the Plymouth Marine Laboratory (Clarke and Warwick, 1998; Clarke and Gorley, 2015). Studies on assessment of assemblages of fish fauna are estimated using Shannon's total diversity index and Pielou's evenness index (Walter and Haynes, 2006; Mallela et al., 2007). The Bray-Curtis similarities were executed to illustrate the similarities between the seasons with a month or season-wise biomass data as an input (Clarke et al., 2008). The Similarity percentage (SIMPER) was computed to find out the contribution of each fish species to find the similarities or dissimilarities within and between the seasons. The species with high contributions i.e., more than $50.00 \%$ was considered as a low cut off point, beyond the cut off points (rare species) were ignored for this analysis (Clarke and Warwick, 2001). The hierarchical agglomerative (dendrogram plot) method was used for clustering using group average linkage for the seasonal bycatch species (Clarke et al., 2008). Linktree was performed using Similarity Percentage analysis to determine the characteristically notably different groups of bycatch fish species on a seasonal basis (Clarke, 1993).

2.5. Classification of trophic level and the habitat of the fish species obtained from the trawl bycatch

The trophic level was estimated by the formula derived by Christensen and Pauly (1992), which is based on the diet composition of the species.

$\mathrm{TROPH}_{i}=1+\sum_{j}^{s} D C_{i j} \times W T P_{j}$

where, $\mathrm{WTP}_{\mathrm{j}}$ represents the fractional trophic level of the prey $\mathrm{j}$, and DCij represents the fraction of $\mathrm{j}$ in the diet of $\mathrm{i}$ and $\mathrm{S}$ is the total number of prey species. In a food web, a primary consumer i.e., herbivore which primarily forages on the plants and detritus, might be in the trophic level values between 2.0 and 2.19. The omnivorous fish which behaves as herbivore and carnivore consuming both plants/detritus and animals may have trophic levels between 2.2 and 2.79 . The secondary and tertiary consumers, which are carnivores, feeding on other animals may have values equal to or greater than 2.8. Values 3.5 to 3.9 are toplevel carnivores, which feed on other secondary carnivores. Above 4.0 are considered as apex predators that feed on other predators (Froese and Pauly, 2018).

Habitat denotes the specific favoured environment preferred by the species. Holthus and Maragos (1995) classified the habitat of marine fishes based on the existence in the water column and feeding behaviour. The classifications were (i) Pelagic (P), primarily occurred in between the depth of 0 and $200 \mathrm{~m}$ in the water column and did not depend on the benthic organisms for feeding; (ii) Benthopelagic (BP), refuges and feeds on or close to the bottom, also in midwater; (iii) Demersal (D), lives and feeds on the bottom; (iv) Reef-associated (RA), shelters and forages on the reef areas; (v) Bathydemersal (BD), lives and feeds on the bottom at below $200 \mathrm{~m}$ depth (Froese and Pauly, 2018). The data and details values on the trophic level, habitat and IUCN criteria were referred from the FishBase website (Froese and Pauly, 2018), where all the trophic level values were estimated by the above formula.

\section{Results}

\subsection{Diversity of ichthyofauna associated with trawl bycatches}

In the present study, a total of 156 species of fishes belonging to two classes, 14 orders, 66 families and 119 genera was recorded from the fish bycatch of trawl net fishery of the Chennai coast. Among these finfish species, bony fishes included 152 species belonging to 12 orders, 62 families, and 115 genera, whereas the cartilaginous fishes included only four species of rays belonging to two orders, four families and four genera. The maximum number of species was recorded in September (112) followed by December (105) and October (104). The minimum number was recorded in June (59) followed by March (88), February (90), and July (90). The season-wise analysis of occurrence data revealed the maximum number of species during South-west monsoon (123) and North-east monsoon (123). The minimum number of species was registered during Post-monsoon (107).

Among the 14 orders, the order Perciformes was observed to contribute $47 \%$ of species recorded with 74 species followed by Scorpaeniformes (12\%; 19 species), Tetraodontiformes (11\%; 18 species), Pleuronectiformes (8\%; 13 species), Syngnathiformes (5\%; 8 species), Anguilliformes (3\%; 4 species), Clupeiformes (3\%; 4 species), Lophiiformes (3\%; 5 species). The order, Aulopiformes and Torpediniformes were observed to contribute each $2 \%$ with 3 species. Meanwhile the order Siluriformes was observed to be represented with only two species while the orders, Beryciformes, Gadiformes and Myliobatiformes each contributed 1\% with one species. The family Leiognathidae was numerically dominant in catch composition and the species included Leiognathus equulus (6\%), Equulites lineolatus (5\%), Gazza achlamys (5\%), Karalla dussumieri (4\%), followed by Otolithes ruber (3\%), Nibea maculata (2\%) belonging to the family Sciaenidae. Bycatch landings consisting of high portions of juveniles of both target and non-target fish species were observed during this entire study. Commercially important species like Caranx ignobilis, Selaroides leptolepis, Pampus argenteus, Lutjanus fulviflamma and Sillago sihama, were noted with sizes less than their length at first maturity (Lm), which shows that trawler exploits most of the fishes that have not attained even $\mathrm{Lm}$. Length class of most of the species were observed with less than $12 \mathrm{~cm}$ (Table 1).

As per the IUCN red list, among these 156 species recorded, 56 species (36\%) were noted to be as Least Concern (LC), 91 species (58\%) as Not Evaluated (NE), seven species (5\%) as Data Deficient (DD) and two species (1\%) as Vulnerable Condition (VU). Three new reports to Indian waters were documented during the study period which includes Pseudorhombus diplospilus (Kodeeswaran et al., 2020a), Myersina yangii (Kodeeswaran et al., 2020b) and Inimicus didactylus (Kodeeswaran et al., 2020c) and two new reports to Chennai coast viz., Scorpaenopsis ramaraoi and Myersina filifer were also documented.

\subsection{Diversity indices of various fishes from trawl bycatch}

The seasonal variation in Shannon-Wiener species diversity (H') was found to range from 5.37 (South-west monsoon) to 5.53 (North-east monsoon) and monthly values of $\mathrm{H}$ ' were estimated to be within the range of 4.53 (June) to 5.63 (December). The seasonal variation in species richness (d) was found to range from 9.77 (Post-monsoon) to 11.51 (South-west monsoon) and monthly values were found to range from 6.33 (June) to 11.65 (September). The seasonal values for Species evenness (J') were observed in the ranges of 0.77 (South-west monsoon) to 0.81 (Post-monsoon) and monthly values were estimated to be within the range of 0.75 (October) to 0.84 (March). The season-wise taxonomic diversity indicated that the South-west monsoon (81.85) 
Table 1

Checklist of fish bycatch of trawl net fishery of Chennai coast including habitat, size range, abundance, IUCN criteria, TrLs, $\mathrm{L}_{\mathrm{m}}$

\begin{tabular}{|c|c|c|c|c|c|c|c|c|c|}
\hline & Order & Family & Species & Habitat & Size $(\mathrm{cm})$ & $\mathrm{L}_{\mathrm{m}}(\mathrm{cm})$ & $\begin{array}{l}\text { Trophic } \\
\text { Level }\end{array}$ & Abundance & $\begin{array}{l}\text { IUCN } \\
\text { Status }\end{array}$ \\
\hline 1. & Anguilliformes & Congridae & $\begin{array}{l}\text { Uroconger lepturus (Richardson, } \\
1845 \text { ) }\end{array}$ & $\mathrm{D}$ & 31.9 & - & 3.5 & $\mathrm{~F}$ & NE \\
\hline 2. & & Muraenidae & Echidna nebulosa (Ahl, 1789) & RA & 24.8 & - & 4.0 & $\mathrm{~F}$ & NE \\
\hline 3. & & & $\begin{array}{l}\text { Gymnothorax javanicus } \\
\text { (Bleeker, 1859) }\end{array}$ & RA & 51.7 & - & 3.9 & $\mathrm{R}$ & $\mathrm{NE}$ \\
\hline 4. & & & $\begin{array}{l}\text { Gymnothorax prolatus Sasaki \& } \\
\text { Amaoka, } 1991\end{array}$ & $\mathrm{D}$ & 28.1 & - & 3.8 & $\mathrm{R}$ & $\mathrm{NE}$ \\
\hline 5. & Aulopiformes & Synodontidae & $\begin{array}{l}\text { Harpadon nehereus (Hamilton, } \\
1822 \text { ) }\end{array}$ & $\mathrm{BP}$ & 15.4 & - & 4.2 & $\mathrm{R}$ & NE \\
\hline 6. & & & Saurida tumbil (Bloch, 1795) & RA & $4.3-12.9$ & 30.0 & 4.4 & $\mathrm{~F}$ & $\mathrm{NE}$ \\
\hline 7. & & & $\begin{array}{l}\text { Trachinocephalus myops } \\
\text { (Forster, 1801) }\end{array}$ & RA & $4.1-23.9$ & - & 4.4 & $\mathrm{~F}$ & LC \\
\hline 8. & Beryciformes & Holocentridae & $\begin{array}{l}\text { Myripristis berndti (Jordan \& } \\
\text { Evermann, 1903) }\end{array}$ & RA & 14.5 & - & 3.7 & M & LC \\
\hline 9. & Clupeiformes & Engraulidae & $\begin{array}{l}\text { Encrasicholina punctifer Fowler, } \\
1938\end{array}$ & RA & $2.7-6.7$ & - & 3.3 & $\mathrm{~F}$ & LC \\
\hline 10. & & & $\begin{array}{l}\text { Stolephorus commersonnii } \\
\text { Lacepède, } 1803\end{array}$ & $\mathrm{P}$ & $3.0-6.1$ & 7.3 & 3.1 & $\mathrm{~F}$ & LC \\
\hline 11. & & & $\begin{array}{l}\text { Stolephorus indicus (van } \\
\text { Hasselt, 1823) }\end{array}$ & $\mathrm{P}$ & $2.9-7.3$ & 9.0 & 3.6 & $\mathrm{~F}$ & LC \\
\hline 12. & & & $\begin{array}{l}\text { Thryssa malabarica (Bloch, } \\
\text { 1795) }\end{array}$ & $\mathrm{P}$ & 16.8 & - & 3.4 & $\mathrm{~F}$ & DD \\
\hline 13. & Gadiformes & $\begin{array}{l}\text { Bregmaceroti- } \\
\text { dae }\end{array}$ & $\begin{array}{l}\text { Bregmaceros mcclellandi } \\
\text { Thompson, } 1840\end{array}$ & $\mathrm{P}$ & $3.2-7.8$ & - & 3.3 & $\mathrm{~F}$ & NE \\
\hline 14. & Lophiiformes & Antennariidae & $\begin{array}{l}\text { Antennatus coccineus (Lesson, } \\
\text { 1831) }\end{array}$ & RA & 9.96 & - & 4.2 & $\mathrm{R}$ & NE \\
\hline 15. & & & $\begin{array}{l}\text { Antennarius indicus Schultz, } \\
1964\end{array}$ & RA & 9.9 & - & 4.3 & $\mathrm{R}$ & $\mathrm{NE}$ \\
\hline 16. & & Lophiidae & $\begin{array}{l}\text { Lophiodes mutilus (Alcock, } \\
\text { 1894) }\end{array}$ & $\mathrm{BD}$ & 8.2 & - & 4.2 & $\mathrm{R}$ & NE \\
\hline 17. & & Ogcocephalidae & Halieutaea stellata (Vahl, 1797) & $\mathrm{D}$ & 12.4 & - & 3.5 & $\mathrm{~F}$ & NE \\
\hline 18. & & & $\begin{array}{l}\text { Halieutaea indica (Annandale \& } \\
\text { Jenkins, 1910) }\end{array}$ & $\mathrm{D}$ & 7.3 & - & 3.4 & $\mathrm{~F}$ & NE \\
\hline 19. & $\begin{array}{l}\text { Myliobati- } \\
\text { formes }\end{array}$ & Dasyatidae & $\begin{array}{l}\text { Brevitrygon imbricata (Bloch \& } \\
\text { Schneider, 1801) }\end{array}$ & $\mathrm{D}$ & $9.1-12.8$ & 19.0 & 3.5 & $\mathrm{~F}$ & DD \\
\hline 20. & Perciformes & Acanthuridae & Acanthurus mata (Cuvier, 1829) & RA & 16.6 & - & 2.2 & $\mathrm{R}$ & LC \\
\hline 21. & & Ammodytidae & $\begin{array}{l}\text { Bleekeria kallolepis Günther, } \\
1862\end{array}$ & $\mathrm{P}$ & 13.3 & - & 3.2 & $\mathrm{R}$ & $\mathrm{NE}$ \\
\hline 22. & & & $\begin{array}{l}\text { Bleekeria murtii Joshi, Zacharia } \\
\text { \& Kanthan, } 2012\end{array}$ & $\mathrm{BP}$ & 11.5 & - & 3.2 & $\mathrm{R}$ & NE \\
\hline 23. & & Apogonidae & $\begin{array}{l}\text { Apogonichthyoides taeniatus } \\
\text { (Cuvier, 1828) }\end{array}$ & RA & 6.4 & - & 3.5 & $\mathrm{R}$ & NE \\
\hline 24. & & & $\begin{array}{l}\text { Archamia bleekeri (Günther, } \\
\text { 1859) }\end{array}$ & RA & 8.2 & - & 3.7 & $\mathrm{~F}$ & NE \\
\hline 25. & Perciformes & & Jaydia ellioti (Day, 1875) & RA & 11.5 & - & 3.6 & $\mathrm{~F}$ & NE \\
\hline 26. & & & $\begin{array}{l}\text { Jaydia striata (Smith and } \\
\text { Radcliffe 1912) }\end{array}$ & RA & 7.0 & - & 3.4 & $\mathrm{R}$ & $\mathrm{NE}$ \\
\hline 27. & & & $\begin{array}{l}\text { Ostorhinchus fasciatus (White, } \\
\text { 1790) }\end{array}$ & $\mathrm{RA}$ & 14.1 & - & 3.6 & $\mathrm{~F}$ & NE \\
\hline 28. & & & $\begin{array}{l}\text { Ostorhinchus oxina (Fraser, } \\
\text { 1999) }\end{array}$ & $\mathrm{D}$ & 8.4 & - & 3.5 & $\mathrm{R}$ & NE \\
\hline 29. & & Blenniidae & Xiphasia setifer Swainson, 1839 & $\mathrm{BP}$ & 31.9 & - & 3.9 & M & LC \\
\hline 30. & & Callionymidae & $\begin{array}{l}\text { Synchiropus lineolatus } \\
\text { (Valenciennes, 1837) }\end{array}$ & $\mathrm{D}$ & 9.4 & - & 3.3 & $\mathrm{~F}$ & NE \\
\hline 31. & & Carangidae & Alectis indica (Rüppell, 1830) & RA & 7.2 & - & 4.1 & $\mathrm{~F}$ & LC \\
\hline 32. & & & Alectis ciliaris (Bloch, 1787) & RA & 6.5 & - & 4.0 & M & LC \\
\hline 33. & & & Alepes kleinii (Bloch, 1793) & RA & $5.5-10.9$ & 12.9 & 3.5 & $\mathrm{~F}$ & $\mathrm{NE}$ \\
\hline 34. & & & Caranx ignobilis (Forsskål, 1775) & RA & $8.3-12.8$ & 60.0 & 4.2 & $\mathrm{~F}$ & LC \\
\hline 35. & & & $\begin{array}{l}\text { Gnathanodon speciosus } \\
\text { (Forsskål, 1775) }\end{array}$ & RA & 3.8 & 32.5 & 3.8 & $\mathrm{~F}$ & LC \\
\hline
\end{tabular}


Table 1 (continued)

\begin{tabular}{|c|c|c|c|c|c|c|c|c|c|}
\hline & Order & Family & Species & Habitat & Size $(\mathrm{cm})$ & $\mathrm{L}_{\mathrm{m}}(\mathrm{cm})$ & $\begin{array}{l}\text { Trophic } \\
\text { Level }\end{array}$ & Abundance & $\begin{array}{l}\text { IUCN } \\
\text { Status }\end{array}$ \\
\hline 36. & & & $\begin{array}{l}\text { Naucrates ductor (Linnaeus, } \\
1758 \text { ) }\end{array}$ & $\mathrm{RA}$ & 6.7 & - & 3.4 & $\mathrm{R}$ & LC \\
\hline 37. & & & $\begin{array}{l}\text { Pampus argenteus (Euphrasen, } \\
\text { 1788) }\end{array}$ & $\mathrm{BP}$ & $5.3-7.9$ & 25.3 & 3.3 & $\mathrm{~F}$ & $\mathrm{NE}$ \\
\hline 38. & & & $\begin{array}{l}\text { Parastromateus niger (Bloch, } \\
1795 \text { ) }\end{array}$ & RA & $4.4-8.1$ & - & 2.9 & $\mathrm{~F}$ & $\mathrm{NE}$ \\
\hline 39. & & & $\begin{array}{l}\text { Selaroides leptolepis (Cuvier, } \\
1833 \text { ) }\end{array}$ & $\mathrm{RA}$ & $6.4-10.9$ & 11.9 & 3.8 & $\mathrm{~F}$ & LC \\
\hline 40. & & Cepolidae & $\begin{array}{l}\text { Acanthocepola abbreviata } \\
\text { (Valenciennes, 1835) }\end{array}$ & $\mathrm{BP}$ & 22.98 & - & 3.4 & $\mathrm{~F}$ & $\mathrm{NE}$ \\
\hline 41. & & & $\begin{array}{l}\text { Acanthocepola indica (Day, } \\
\text { 1888) }\end{array}$ & $\mathrm{BP}$ & 21.1 & - & 3.4 & $\mathrm{~F}$ & $\mathrm{NE}$ \\
\hline 42. & & & $\begin{array}{l}\text { Acanthocepola limbata } \\
\text { (Valenciennes, 1835) }\end{array}$ & $\mathrm{D}$ & 12.7 & - & 3.4 & $\mathrm{R}$ & NE \\
\hline 43. & & Chaetodontidae & $\begin{array}{l}\text { Chaetodon decussatus Cuvier, } \\
1829\end{array}$ & $\mathrm{RA}$ & 9.7 & - & 2.6 & M & LC \\
\hline 44. & & Ephippidae & Ephippus orbis (Bloch, 1787) & RA & 6.6 & - & 4.0 & $\mathrm{~F}$ & $\mathrm{NE}$ \\
\hline 45. & & Gerreidae & Gerres erythrourus (Bloch, 1791) & RA & $7.2-11.0$ & - & 3.3 & $\mathrm{~F}$ & LC \\
\hline 46. & & & $\begin{array}{l}\text { Gerres filamentosus Cuvier, } \\
1829\end{array}$ & $\mathrm{D}$ & $7.4-11.8$ & 19.0 & 3.3 & $\mathrm{~F}$ & $\mathrm{LC}$ \\
\hline 47. & & Gobiidae & $\begin{array}{l}\text { Arcygobius baliurus } \\
\text { (Valenciennes, 1837) }\end{array}$ & $\mathrm{D}$ & $3.4-8.9$ & - & 3.4 & $\mathrm{~F}$ & DD \\
\hline 48. & & & $\begin{array}{l}\text { Myersina filifer (Valenciennes, } \\
\text { 1837) }\end{array}$ & $\mathrm{D}$ & 9.2 & - & 3.4 & $\mathrm{R}$ & LC \\
\hline 49. & & & Myersina yangii (Chen, 1960) & $\mathrm{D}$ & 7.8 & - & 3.3 & $\mathrm{R}$ & NE \\
\hline 50. & & & $\begin{array}{l}\text { Oxyurichthys auchenolepis } \\
\text { Bleeker, } 1876\end{array}$ & $\mathrm{D}$ & 7.9 & - & 3.7 & $\mathrm{R}$ & $\mathrm{NE}$ \\
\hline 51. & & & $\begin{array}{l}\text { Parachaeturichthys polynema } \\
\text { (Bleeker, 1853) }\end{array}$ & $\mathrm{D}$ & $3.2-13.9$ & - & 3.1 & $\mathrm{~F}$ & NE \\
\hline 52. & Perciformes & & $\begin{array}{l}\text { Taenioides anguillaris (Linnaeus, } \\
\text { 1758) }\end{array}$ & $\mathrm{D}$ & 29.7 & - & 4.0 & $\mathrm{R}$ & NE \\
\hline 53. & & & $\begin{array}{l}\text { Taenioides cf. esquivel Smith, } \\
1947\end{array}$ & $\mathrm{D}$ & 24.9 & - & 3.9 & $\mathrm{R}$ & $\mathrm{NE}$ \\
\hline 54. & & & $\begin{array}{l}\text { Trypauchen vagina (Bloch \& } \\
\text { Schneider, 1801) }\end{array}$ & $\mathrm{D}$ & 16.6 & - & 3.5 & $\mathrm{~F}$ & NE \\
\hline 55. & & Haemulidae & $\begin{array}{l}\text { Diagramma pictum (Thunberg, } \\
\text { 1792) }\end{array}$ & $\mathrm{RA}$ & 11.5 & 31.8 & 3.7 & $\mathrm{~F}$ & $\mathrm{NE}$ \\
\hline 56. & & & $\begin{array}{l}\text { Pomadasys maculatus (Bloch, } \\
\text { 1793) }\end{array}$ & $\mathrm{RA}$ & 10.8 & - & 4.0 & $\mathrm{~F}$ & $\mathrm{NE}$ \\
\hline 57. & & Labridae & $\begin{array}{l}\text { Iniistius bimaculatus (Rüppell, } \\
\text { 1829) }\end{array}$ & $\mathrm{D}$ & 17.4 & - & 3.5 & $\mathrm{R}$ & LC \\
\hline 58. & & & $\begin{array}{l}\text { Xyrichtys novacula (Linnaeus, } \\
1758 \text { ) }\end{array}$ & RA & 13.7 & - & 3.5 & $\mathrm{R}$ & LC \\
\hline 59. & & Leiognathidae & $\begin{array}{l}\text { Equulites lineolatus } \\
\text { (Valenciennes, 1835) }\end{array}$ & $\mathrm{D}$ & 7.8 & - & 3.5 & $\mathrm{~F}$ & NE \\
\hline 60. & & & $\begin{array}{l}\text { Gazza achlamys Jordan \& } \\
\text { Starks, } 1917\end{array}$ & RA & $5.1-6.9$ & - & 3.7 & $\mathrm{~F}$ & LC \\
\hline 61. & & & $\begin{array}{l}\text { Karalla dussumieri } \\
\text { (Valenciennes, 1835) }\end{array}$ & $\mathrm{D}$ & $5.4-8.7$ & - & 3.2 & $\mathrm{~F}$ & $\mathrm{NE}$ \\
\hline 62. & & & $\begin{array}{l}\text { Leiognathus equulus (Forsskål, } \\
1775 \text { ) }\end{array}$ & $\mathrm{D}$ & $3.0-6.9$ & 10.7 & 3.0 & $\mathrm{~F}$ & LC \\
\hline 63. & & & $\begin{array}{l}\text { Nuchequula blochii } \\
\text { (Valenciennes, 1835) }\end{array}$ & $\mathrm{D}$ & $4.1-6.8$ & - & 2.9 & $\mathrm{~F}$ & $\mathrm{NE}$ \\
\hline 64. & & & Secutor insidiator (Bloch, 1787) & $\mathrm{D}$ & 7.7 & 7.5 & 2.8 & $\mathrm{~F}$ & $\mathrm{NE}$ \\
\hline 65. & & Lutjanidae & $\begin{array}{l}\text { Lutjanus fulviflamma (Forsskål, } \\
\text { 1775) }\end{array}$ & $\mathrm{RA}$ & $5.1-11.2$ & 17.1 & 3.8 & $\mathrm{~F}$ & $\mathrm{NE}$ \\
\hline 66. & & & $\begin{array}{l}\text { Lutjanus quinquelineatus (Bloch, } \\
1790 \text { ) }\end{array}$ & $\mathrm{RA}$ & 8.9 & - & 3.7 & M & $\mathrm{NE}$ \\
\hline 67. & & Menidae & $\begin{array}{l}\text { Mene maculata (Bloch \& } \\
\text { Schneider, 1801) }\end{array}$ & RA & $3.6-7.2$ & 14 & 3.5 & M & $\mathrm{NE}$ \\
\hline 68. & & Microdesmidae & $\begin{array}{l}\text { Ptereleotris cf. arabica Randall } \\
\text { \& Hoese, } 1985\end{array}$ & $\mathrm{D}$ & 13.3 & - & 3.4 & $\mathrm{R}$ & $\mathrm{NE}$ \\
\hline
\end{tabular}


Table 1 (continued)

\begin{tabular}{|c|c|c|c|c|c|c|c|c|c|}
\hline & Order & Family & Species & Habitat & Size $(\mathrm{cm})$ & $\mathrm{L}_{\mathrm{m}}(\mathrm{cm})$ & $\begin{array}{l}\text { Trophic } \\
\text { Level }\end{array}$ & Abundance & $\begin{array}{l}\text { IUCN } \\
\text { Status }\end{array}$ \\
\hline 69. & & Mullidae & $\begin{array}{l}\text { Upeneus vittatus (Forsskål, } \\
\text { 1775) }\end{array}$ & RA & $8.7-16.3$ & - & 3.6 & $\mathrm{~F}$ & LC \\
\hline 70. & & Nemipteridae & Scolopsis vosmeri (Bloch, 1792) & RA & 8.9 & - & 3.5 & M & NE \\
\hline 71. & & & $\begin{array}{l}\text { Nemipterus japonicus (Bloch, } \\
\text { 1791) }\end{array}$ & $\mathrm{D}$ & $6.6-11.8$ & 14 & 4.1 & $\mathrm{~F}$ & $\mathrm{NE}$ \\
\hline 72. & & & $\begin{array}{l}\text { Nemipterus nematophorus } \\
\text { (Bleeker, 1854) }\end{array}$ & $\mathrm{D}$ & $6.7-10.9$ & - & 3.8 & $\mathrm{~F}$ & NE \\
\hline 73. & & $\begin{array}{l}\text { Opistognathi- } \\
\text { dae }\end{array}$ & $\begin{array}{l}\text { Opistognathus nigromarginatus } \\
\text { (Rüppell, 1830) }\end{array}$ & $\mathrm{D}$ & 15.327 & - & 3.6 & $\mathrm{R}$ & $\mathrm{NE}$ \\
\hline 74. & & $\begin{array}{l}\text { Opistognathi- } \\
\text { dae }\end{array}$ & $\begin{array}{l}\text { Opistognathus rosenbergii } \\
\text { Bleeker, } 1856\end{array}$ & RA & 12 & - & 3.5 & $\mathrm{R}$ & $\mathrm{NE}$ \\
\hline 75. & & & $\begin{array}{l}\text { Opistognathus macrolepis Peters, } \\
1866\end{array}$ & RA & 6.3 & - & 3.6 & $\mathrm{R}$ & $\mathrm{NE}$ \\
\hline 76. & & Pinguipedidae & $\begin{array}{l}\text { Parapercis alboguttata (Günther, } \\
\text { 1872) }\end{array}$ & $\mathrm{D}$ & $5.7-11.2$ & - & 3.6 & $\mathrm{~F}$ & $\mathrm{NE}$ \\
\hline 77. & & Pomacentridae & $\begin{array}{l}\text { Neopomacentrus cyanomos } \\
\text { (Bleeker, 1856) }\end{array}$ & RA & 9.2 & - & 3.4 & $\mathrm{R}$ & $\mathrm{NE}$ \\
\hline 78. & & Rachycentridae & $\begin{array}{l}\text { Rachycentron canadum } \\
\text { (Linnaeus, 1766) }\end{array}$ & RA & 21.3 & 63.0 & 4.0 & $\mathrm{R}$ & LC \\
\hline 79. & & Sciaenidae & $\begin{array}{l}\text { Johnius dussumieri (Cuvier, } \\
\text { 1830) }\end{array}$ & $\mathrm{D}$ & $5.9-12.8$ & 11.5 & 4.1 & $\mathrm{~F}$ & $\mathrm{NE}$ \\
\hline 80. & Perciformes & & $\begin{array}{l}\text { Nibea maculata (Bloch \& } \\
\text { Schneider, 1801) }\end{array}$ & $\mathrm{D}$ & $6.0-11.7$ & - & 3.6 & $\mathrm{~F}$ & $\mathrm{NE}$ \\
\hline 81. & & & $\begin{array}{l}\text { Otolithes ruber (Bloch \& } \\
\text { Schneider, 1801) }\end{array}$ & $\mathrm{BP}$ & $6.3-15.5$ & 22.6 & 3.6 & $\mathrm{~F}$ & $\mathrm{NE}$ \\
\hline 82. & & Serranidae & $\begin{array}{l}\text { Epinephelus radiatus (Day, } \\
1868 \text { ) }\end{array}$ & $\mathrm{D}$ & 7.7 & - & 4.0 & $\mathrm{R}$ & LC \\
\hline 83. & & Siganidae & $\begin{array}{l}\text { Siganus canaliculatus (Park, } \\
\text { 1797) }\end{array}$ & RA & $5.6-10.3$ & 11.6 & 2.8 & $\mathrm{~F}$ & LC \\
\hline 84. & & & $\begin{array}{l}\text { Siganus lineatus (Valenciennes, } \\
\text { 1835) }\end{array}$ & RA & $5.9-10.4$ & - & 2.0 & M & LC \\
\hline 85. & & Sillaginidae & Sillago sihama (Forsskål, 1775) & RA & $6.9-9.1$ & 22.5 & 3.3 & M & LC \\
\hline 86. & & & Sillago vincenti (McKay, 1980) & $\mathrm{D}$ & $6.2-8.9$ & - & 3.3 & M & LC \\
\hline 87. & & Sphyraenidae & Sphyraena jello Cuvier, 1829 & RA & $7.6-10.4$ & - & 4.5 & M & NE \\
\hline 88. & & Terapontidae & Terapon jarbua (Forsskål, 1775) & $\mathrm{D}$ & $5.4-11.0$ & 13.0 & 3.9 & $\mathrm{~F}$ & LC \\
\hline 89. & & Terapontidae & Terapon theraps Cuvier, 1829 & RA & 7.8 & - & 3.5 & M & LC \\
\hline 90. & & Trichiuridae & $\begin{array}{l}\text { Trichiurus lepturus Linnaeus, } \\
1758\end{array}$ & $\mathrm{BP}$ & $9.1-21.3$ & 50.6 & 4.4 & $\mathrm{~F}$ & LC \\
\hline 91. & & Trichonotidae & $\begin{array}{l}\text { Trichonotus cyclograptus (Alock, } \\
1890 \text { ) }\end{array}$ & $\mathrm{D}$ & 13.7 & - & 3.4 & $\mathrm{R}$ & $\mathrm{NE}$ \\
\hline 92. & & Uranoscopidae & $\begin{array}{l}\text { Ichthyscopus lebeck ( Bloch and } \\
\text { Schneider, 1801) }\end{array}$ & $\mathrm{D}$ & 13.2 & - & 4.5 & M & $\mathrm{NE}$ \\
\hline 93. & & & $\begin{array}{l}\text { Uranoscopus guttatus Cuvier, } \\
1829\end{array}$ & $\mathrm{D}$ & 9.2 & - & 4.2 & $\mathrm{R}$ & NE \\
\hline 94. & $\begin{array}{l}\text { Pleuronecti- } \\
\text { formes }\end{array}$ & Bothidae & $\begin{array}{l}\text { Arnoglossus tapeinosoma } \\
\text { (Bleeker, 1865) }\end{array}$ & $\mathrm{D}$ & $6.3-11.7$ & - & 3.5 & M & $\mathrm{NE}$ \\
\hline 95. & & & $\begin{array}{l}\text { Bothus myriaster (Temminck \& } \\
\text { Schlegel, 1846) }\end{array}$ & $\mathrm{D}$ & $5.4-9.7$ & - & 3.5 & $\mathrm{~F}$ & $\mathrm{NE}$ \\
\hline 96. & & & $\begin{array}{l}\text { Crossorhombus azureus (Alcock, } \\
\text { 1889) }\end{array}$ & $\mathrm{D}$ & 12.3 & - & 3.5 & M & NE \\
\hline 97. & & & $\begin{array}{l}\text { Grammatobothus } \\
\text { polyophthalmus (Bleeker, 1865) }\end{array}$ & $\mathrm{D}$ & 9.7 & - & 3.6 & $\mathrm{R}$ & LC \\
\hline 98. & & Paralichthyidae & $\begin{array}{l}\text { Pseudorhombus argus Weber, } \\
1913\end{array}$ & $\mathrm{D}$ & 12.1 & - & 3.5 & $\mathrm{R}$ & $\mathrm{NE}$ \\
\hline 99. & & & $\begin{array}{l}\text { Pseudorhombus diplospilus } \\
\text { (Norman, 1926) }\end{array}$ & $\mathrm{D}$ & 13.3 & - & 3.5 & $\mathrm{R}$ & $\mathrm{NE}$ \\
\hline 100. & & & $\begin{array}{l}\text { Pseudorhombus elevatus Ogilby, } \\
1912\end{array}$ & $\mathrm{D}$ & 11.7 & - & 3.5 & M & $\mathrm{NE}$ \\
\hline 101. & & & $\begin{array}{l}\text { Pseudorhombus triocellatus } \\
\text { (Bloch \& Schneider, 1801) }\end{array}$ & $\mathrm{D}$ & $6.3-9.2$ & - & 3.5 & $\mathrm{~F}$ & $\mathrm{NE}$ \\
\hline 102. & & Psettodidae & $\begin{array}{l}\text { Psettodes erumei (Bloch \& } \\
\text { Schneider, 1801) }\end{array}$ & $\mathrm{D}$ & $5.5-13.4$ & - & 4.4 & M & $\mathrm{NE}$ \\
\hline
\end{tabular}


Table 1 (continued)

\begin{tabular}{|c|c|c|c|c|c|c|c|c|c|}
\hline & Order & Family & Species & Habitat & Size $(\mathrm{cm})$ & $\mathrm{L}_{\mathrm{m}}(\mathrm{cm})$ & $\begin{array}{l}\text { Trophic } \\
\text { Level }\end{array}$ & Abundance & $\begin{array}{l}\text { IUCN } \\
\text { Status }\end{array}$ \\
\hline 103. & & Soleidae & $\begin{array}{l}\text { Heteromycteris oculus (Alcock, } \\
\text { 1889) }\end{array}$ & $\mathrm{D}$ & $6.8-12.9$ & - & 3.4 & $\mathrm{~F}$ & $\mathrm{NE}$ \\
\hline 104. & & Soleidae & $\begin{array}{l}\text { Synaptura albomaculata Kaup, } \\
1858\end{array}$ & $\mathrm{D}$ & 7.4-13.6 & - & 3.5 & M & NE \\
\hline 105. & & & Zebrias quagga (Kaup, 1858) & $\mathrm{D}$ & $6.6-10.4$ & - & 3.5 & $\mathrm{~F}$ & $\mathrm{NE}$ \\
\hline 106. & & & $\begin{array}{l}\text { Zebrias synapturoides (Jenkins, } \\
1910 \text { ) }\end{array}$ & $\mathrm{D}$ & $6.8-11.7$ & - & 3.5 & $\mathrm{~F}$ & $\mathrm{NE}$ \\
\hline 107. & $\begin{array}{l}\text { Scorpaeni- } \\
\text { formes }\end{array}$ & Apistidae & $\begin{array}{l}\text { Apistus carinatus (Bloch \& } \\
\text { Schneider, 1801) }\end{array}$ & $\mathrm{D}$ & 8.7 & - & 3.5 & M & $\mathrm{NE}$ \\
\hline 108. & & Aploactinidae & Cocotropus roseus Day, 1875 & $\mathrm{D}$ & $2.6-6.76$ & - & 3.2 & $\mathrm{R}$ & $\mathrm{NE}$ \\
\hline 109. & & Dactylopteridae & $\begin{array}{l}\text { Dactyloptena papilio (Ogilby, } \\
1910 \text { ) }\end{array}$ & $\mathrm{D}$ & $5.9-12.6$ & - & 3.5 & $\mathrm{R}$ & $\mathrm{NE}$ \\
\hline 110. & & & $\begin{array}{l}\text { Dactyloptena macracantha } \\
\text { (Bleeker, 1855) }\end{array}$ & $\mathrm{D}$ & 15.4 & - & 3.5 & $\mathrm{R}$ & NE \\
\hline 111. & & Platycephalidae & $\begin{array}{l}\text { Grammoplites scaber (Linnaeus, } \\
1758 \text { ) }\end{array}$ & $\mathrm{D}$ & $4.9-8.7$ & - & 3.8 & $\mathrm{~F}$ & $\mathrm{NE}$ \\
\hline 112. & & & $\begin{array}{l}\text { Grammoplites suppositus } \\
\text { (Troschel, 1840) }\end{array}$ & $\mathrm{D}$ & $4.2-11.8$ & - & 3.8 & $\mathrm{~F}$ & LC \\
\hline 113. & & & $\begin{array}{l}\text { Platycephalus indicus (Linnaeus, } \\
1758 \text { ) }\end{array}$ & RA & $6.1-24.1$ & 40.0 & 3.6 & $\mathrm{~F}$ & DD \\
\hline 114. & & & Rogadius asper (Cuvier, 1829) & $\mathrm{D}$ & $5-21.7$ & - & 3.6 & $\mathrm{~F}$ & LC \\
\hline 115. & & Scorpaenidae & $\begin{array}{l}\text { Dendrochirus bellus (Jordan \& } \\
\text { Hubbs, 1925) }\end{array}$ & $\mathrm{D}$ & 7.7 & - & 3.8 & M & LC \\
\hline 116. & & & $\begin{array}{l}\text { Dendrochirus brachypterus } \\
\text { (Cuvier, 1829) }\end{array}$ & $\mathrm{RA}$ & 8.4 & - & 3.6 & M & LC \\
\hline 117. & & & $\begin{array}{l}\text { Pteroidichthys amboinensis } \\
\text { Bleeker, } 1856\end{array}$ & $\mathrm{D}$ & 4.9 & - & 3.6 & $\mathrm{R}$ & LC \\
\hline 118. & & & Pterois volitans (Linnaeus, 1758) & RA & $10.8-21$ & & 4.4 & M & LC \\
\hline 119. & $\begin{array}{l}\text { Scorpaeni- } \\
\text { formes }\end{array}$ & Scorpaenidae & $\begin{array}{l}\text { Scorpaenopsis ramaraoi Randall } \\
\text { \& Eschmeyer, } 2001\end{array}$ & $\mathrm{D}$ & 12.9 & - & 4.1 & $\mathrm{R}$ & $\mathrm{LC}$ \\
\hline 120. & & Synanceiidae & $\begin{array}{l}\text { Choridactylus multibarbus } \\
\text { Richardson, } 1848\end{array}$ & $\mathrm{D}$ & $4.1-8.3$ & - & 3.8 & $\mathrm{~F}$ & $\mathrm{NE}$ \\
\hline 121. & & & $\begin{array}{l}\text { Inimicus didactylus (Pallas, } \\
\text { 1769) }\end{array}$ & RA & 12.2 & - & 4.0 & $\mathrm{R}$ & NE \\
\hline 122. & & & $\begin{array}{l}\text { Inimicus sinensis (Valenciennes, } \\
1833 \text { ) }\end{array}$ & RA & 13.7 & - & 4.1 & $\mathrm{R}$ & $\mathrm{NE}$ \\
\hline 123. & & & $\begin{array}{l}\text { Minous dempsterae Eschmeyer, } \\
\text { Hallacher \& Rama-Rao, } 1979\end{array}$ & $\mathrm{D}$ & $4.2-8.7$ & - & 3.8 & $\mathrm{~F}$ & LC \\
\hline 124. & & & $\begin{array}{l}\text { Minous monodactylus (Bloch \& } \\
\text { Schneider, 1801) }\end{array}$ & $\mathrm{D}$ & $4-8.9$ & - & 3.8 & $\mathrm{~F}$ & $\mathrm{NE}$ \\
\hline 125. & & & $\begin{array}{l}\text { Trachicephalus uranoscopus } \\
\text { (Bloch \& Schneider, 1801) }\end{array}$ & $\mathrm{D}$ & 15.5 & - & 3.6 & $\mathrm{~F}$ & $\mathrm{NE}$ \\
\hline 126. & Siluriformes & Plotosidae & $\begin{array}{l}\text { Plotosus lineatus (Thunberg, } \\
\text { 1787) }\end{array}$ & RA & 14.4 & 14.0 & 3.6 & M & $\mathrm{NE}$ \\
\hline 127. & & Ariidae & $\begin{array}{l}\text { Netuma thalassina (Rüppell, } \\
\text { 1837) }\end{array}$ & $\mathrm{D}$ & 12.4 & 36.0 & 3.5 & M & $\mathrm{NE}$ \\
\hline 128. & $\begin{array}{l}\text { Syngnathi- } \\
\text { formes }\end{array}$ & Fistulariidae & $\begin{array}{l}\text { Fistularia commersonii Rüppell, } \\
1838\end{array}$ & RA & $8-14$ & - & 4.3 & $\mathrm{~F}$ & LC \\
\hline 129. & & & $\begin{array}{l}\text { Fistularia petimba Lacepède, } \\
1803\end{array}$ & RA & $9-16.2$ & - & 4.4 & $\mathrm{~F}$ & LC \\
\hline 130. & & Syngnathidae & $\begin{array}{l}\text { Hippocampus kuda Bleeker, } \\
1852\end{array}$ & RA & 7.9 & 14.0 & 3.6 & $\mathrm{R}$ & VU \\
\hline 131. & & & $\begin{array}{l}\text { Hippocampus spinosissimus } \\
\text { (Weber, 1913) }\end{array}$ & RA & 7.3 & 10.4 & 3.4 & $\mathrm{R}$ & VU \\
\hline 132. & & & $\begin{array}{l}\text { Trachyrhamphus bicoarctatus } \\
\text { (Bleeker, 1857) }\end{array}$ & RA & 17.7 & - & 3.8 & $\mathrm{R}$ & LC \\
\hline 133. & $\begin{array}{l}\text { Syngnathi- } \\
\text { formes }\end{array}$ & & $\begin{array}{l}\text { Trachyrhamphus serratus } \\
\text { (Temminck \& Schlegel, 1850) }\end{array}$ & RA & 11.9 & - & 3.7 & $\mathrm{R}$ & $\mathrm{DD}$ \\
\hline 134. & & Syngnathidae & $\begin{array}{l}\text { Trachyrhamphus longirostris } \\
\text { Kaup, } 1856\end{array}$ & D & 12.8 & - & 3.8 & $\mathrm{R}$ & LC \\
\hline 135. & & Centriscidae & $\begin{array}{l}\text { Centriscus scutatus Lin naeus, } \\
1758\end{array}$ & RA & $8.7-13.8$ & - & 3.3 & M & LC \\
\hline
\end{tabular}


Table 1 (continued)

\begin{tabular}{|c|c|c|c|c|c|c|c|c|c|}
\hline & Order & Family & Species & Habitat & Size $(\mathrm{cm})$ & $\mathrm{L}_{\mathrm{m}}(\mathrm{cm})$ & $\begin{array}{l}\text { Trophic } \\
\text { Level }\end{array}$ & Abundance & $\begin{array}{l}\text { IUCN } \\
\text { Status }\end{array}$ \\
\hline 136. & $\begin{array}{l}\text { Tetraodontif- } \\
\text { ormes }\end{array}$ & Balistidae & $\begin{array}{l}\text { Abalistes stellatus (Anonymous, } \\
1798 \text { ) }\end{array}$ & RA & 8.9 & - & 3.4 & M & $\mathrm{NE}$ \\
\hline 137. & & Diodontidae & $\begin{array}{l}\text { Cyclichthys orbicularis (Bloch, } \\
1785 \text { ) }\end{array}$ & RA & 16.9 & - & 3.6 & $\mathrm{~F}$ & NE \\
\hline 138. & & & $\begin{array}{l}\text { Cyclichthys spilostylus (Leis \& } \\
\text { Randall, 1982) }\end{array}$ & RA & 23.7 & - & 3.5 & $\mathrm{R}$ & NE \\
\hline 139. & & & Diodon liturosus Shaw, 1804 & RA & 24.8 & - & 3.5 & $\mathrm{R}$ & NE \\
\hline 140. & & Monacanthidae & $\begin{array}{l}\text { Aluterus monoceros (Linnaeus, } \\
\text { 1758) }\end{array}$ & RA & 9.3 & - & 3.8 & $\mathrm{R}$ & LC \\
\hline 141. & & & $\begin{array}{l}\text { Paramonacanthus frenatus ( } \\
\text { Peters, 1855) }\end{array}$ & $\mathrm{D}$ & 8.5 & - & 3.1 & $\mathrm{~F}$ & NE \\
\hline 142. & & Ostraciidae & $\begin{array}{l}\text { Lactoria cornuta (Linnaeus, } \\
1758 \text { ) }\end{array}$ & RA & 32.45 & - & 3.5 & $\mathrm{R}$ & NE \\
\hline 143. & & & $\begin{array}{l}\text { Tetrosomus gibbosus (Linnaeus, } \\
\text { 1758) }\end{array}$ & $\mathrm{RA}$ & 8.9 & - & 3.5 & $\mathrm{R}$ & LC \\
\hline 144. & & Tetraodontidae & $\begin{array}{l}\text { Arothron hispidus (Linnaeus } \\
\text { 1758) }\end{array}$ & RA & 25.09 & - & 3.2 & $\mathrm{R}$ & LC \\
\hline 145. & & & $\begin{array}{l}\text { Arothron immaculates ((Bloch } \\
\text { and Schneider, 1801) }\end{array}$ & RA & 20.25 & - & 2.5 & $\mathrm{R}$ & LC \\
\hline 146. & & & $\begin{array}{l}\text { Arothron reticularis (Bloch \& } \\
\text { Schneider, 1801) }\end{array}$ & RA & 6.91 & - & 3.4 & $\mathrm{R}$ & LC \\
\hline 147. & & & $\begin{array}{l}\text { Arothron stellatus (Anonymous, } \\
1798 \text { ) }\end{array}$ & $\mathrm{RA}$ & 16.18 & - & 3.7 & $\mathrm{~F}$ & LC \\
\hline 148. & & & $\begin{array}{l}\text { Canthigaster petersii (Bianconi, } \\
\text { 1854) }\end{array}$ & RA & 6.68 & - & 3.1 & $\mathrm{R}$ & LC \\
\hline 149. & & Tetraodontidae & $\begin{array}{l}\text { Chelonodon patoca (Hamilton, } \\
\text { 1822) }\end{array}$ & RA & $17.9-10.6$ & - & 3.1 & $\mathrm{~F}$ & LC \\
\hline 150. & & & $\begin{array}{l}\text { Lagocephalus suezensis (Clark \& } \\
\text { Gohar, 1953) }\end{array}$ & $\mathrm{D}$ & 15.38 & - & 3.5 & $\mathrm{~F}$ & LC \\
\hline 151. & & & Takifugu oblongus (Bloch, 1786) & $\mathrm{D}$ & 16.33 & - & 3.2 & $\mathrm{R}$ & LC \\
\hline 152. & & & $\begin{array}{l}\text { Torquigener brevipinnis (Regan, } \\
\text { 1903) }\end{array}$ & $\mathrm{D}$ & 9.81 & - & 3.3 & $\mathrm{~F}$ & LC \\
\hline 153. & & Tricanthidae & $\begin{array}{l}\text { Tricanthus biaculeatus (Bloch, } \\
1786 \text { ) }\end{array}$ & $\mathrm{D}$ & 18.1 & - & 2.8 & $\mathrm{~F}$ & NE \\
\hline 154. & $\begin{array}{l}\text { Torpedini- } \\
\text { formes }\end{array}$ & Narcinidae & $\begin{array}{l}\text { Narcine brunnea (Annandale, } \\
1909 \text { ) }\end{array}$ & $\mathrm{D}$ & 12.5 & - & 3.1 & $\mathrm{~F}$ & NE \\
\hline 155. & & Narkidae & $\begin{array}{l}\text { Narke dipterygia (Bloch and } \\
\text { Schneider, 1801) }\end{array}$ & $\mathrm{D}$ & 17 & - & 3.2 & $\mathrm{~F}$ & DD \\
\hline 156. & & Torpedinidae & $\begin{array}{l}\text { Torpedo sinuspersici (Olfers, } \\
\text { 1831) }\end{array}$ & RA & $5.6-12.8$ & - & 4.5 & $\mathrm{~F}$ & DD \\
\hline
\end{tabular}

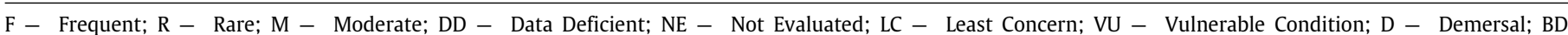
- Bathydemersal; RA - Reef-associated; P - Pelagic; BP - Benthopelagic.
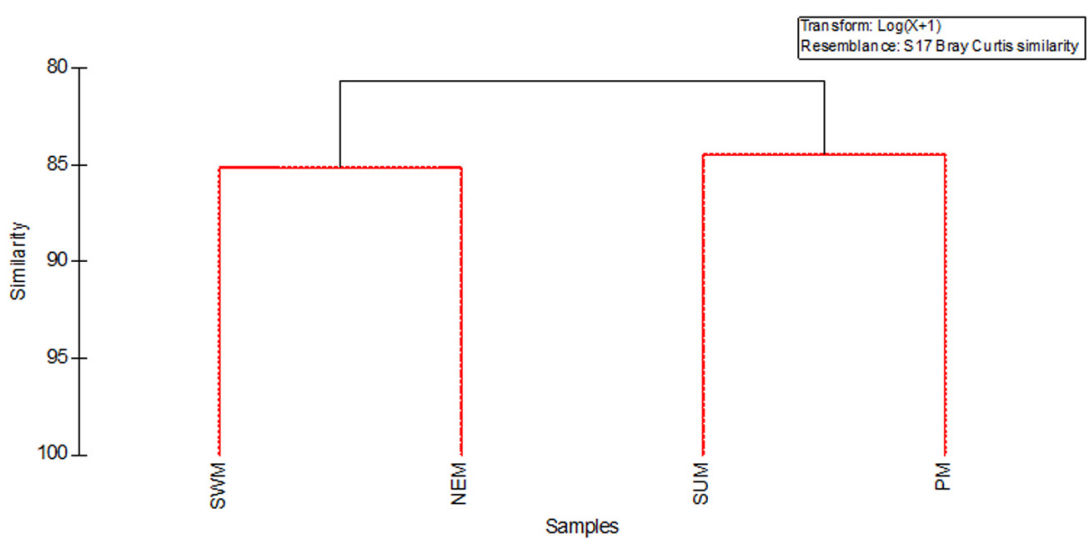

Fig. 2. Dendrogram showing similarities in species composition of fish fauna in bycatch of trawl net fishery of Chennai coast in various seasons.

had the lowest while the Post-monsoon (86.08) had the highest taxonomic diversity. The monthly variation indicates that the lowest values were observed in June 2018 (71.12) and the highest taxonomic diversity was noticed in December 2018 (87.68). The seasonal variation in the taxonomic distinctness $\left(\Delta^{*}\right)$ was found to be in the range of 85.30 (Summer) to 89.24 (Post-monsoon) 


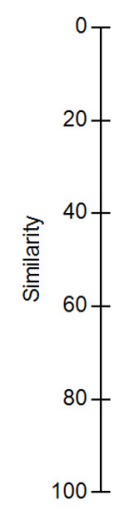

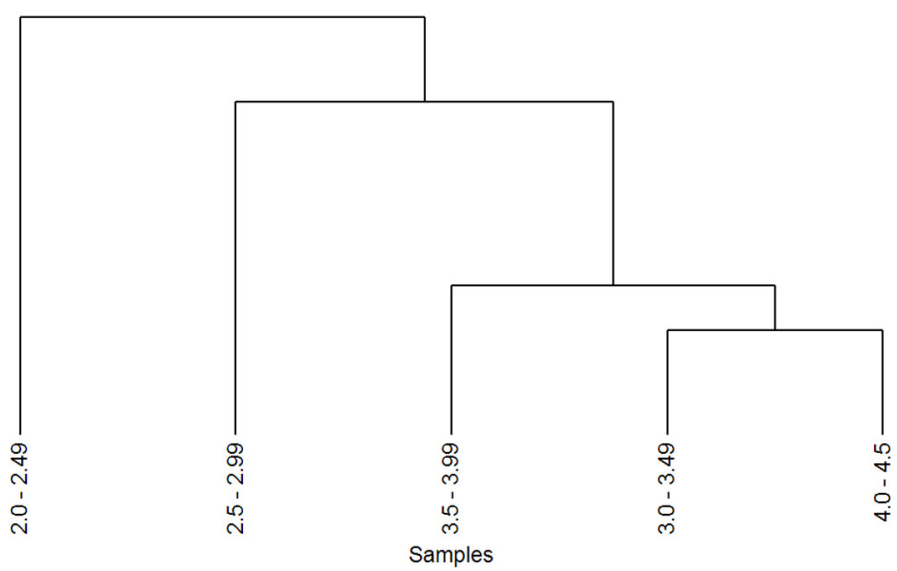

Fig. 3. Dendrogram showing similarities in species composition of Trophic level of fish fauna in bycatch of trawl net fishery of Chennai coast.

Table 2

Seasonal diversity indices of fish fauna in bycatch of trawl net fishery of Chennai coast.

\begin{tabular}{llllllll}
\hline Seasons & $d$ & $J^{\prime}$ & $\mathrm{H}^{\prime}$ & $\Delta$ & $\Delta^{+}$ & $\Lambda^{+}$ & sPhi+ \\
\hline SUMMER & 10.4861 & 0.7992 & 5.4505 & 85.3664 & 92.1342 & 170.331 & 6775 \\
SWM & 11.5062 & 0.7741 & 5.3744 & 81.8524 & 93.2460 & 164.716 & 7075 \\
NEM & 11.2725 & 0.7958 & 5.5250 & 83.9843 & 91.8233 & 176.710 & 7075 \\
PM & 9.7678 & 0.8077 & 5.4452 & 86.0807 & 92.4132 & 167.819 & 6525
\end{tabular}

SWM- South-west Monsoon; NEM- North-east Monsoon; PM- Post-monsoon. $d$ - Margalef"s species richness; J'- Pielous's evenness; H'- Shannon-Wiener species diversity; $\Delta$ - taxonomic diversity; $\Delta^{+-}$Average taxonomic distinctness index; $\Lambda^{+-}$Variation in taxonomic distinctness index; sPhi+- Total phylogenetic diversity.

and monthly $\Delta^{*}$ was estimated to be in the range of 76.71 (June) to 90.73 (April). The estimated seasonal average taxonomic distinctness index $\left(\Delta^{+}\right)$was found to be within the range of 91.82 (North-east monsoon) to 93.25 (South-west monsoon) and monthly values were found to be in the range of 89.51 (June) to 92.77 (September). The season-wise variation in taxonomic distinctness index $(/+)$ was found to be within the range of 164.71 (South-west monsoon) to 176.71 (North-east monsoon) and the month-wise estimated values were found to be within the range of 168.66 (September) to 207.73 (June). The seasonal variation in the total phylogenetic diversity ( $\mathrm{sPhi}+$ ) was estimated and the values were found to be in the range of 6525 (Postmonsoon) to 7075 (South-west and North-east monsoon) and monthly variation values were found to be in the range of 3750 (June) to 6500 (September) (Table 2). The analysis of relative abundance for species revealed that the maximum abundance was during South-west monsoon with $59.83 \%$ of total species followed by North-east monsoon (59.19\%), Post-monsoon (51.28\%) and Summer (50.21\%).

\subsection{Bray-Curtis similarity, dendrogram plot with SIMPER analysis and dominance plot}

The month-wise Bray-Curtis similarity of bycatch ichthyofauna reveals that February and March had a higher similarity with 83.5\% meanwhile, the lower similarity was seen between April and June with 63\%. In the dendrogram plot, the South-west and North-east monsoon shows the maximum similarity of about $85 \%$, which links with another group of Summer and Post-monsoon at $84 \%$ similarity (Fig. 2). The K-Dominance curve that represented December which was at the lower side of the plot shows more fish diversity and less dominance whereas June month is at the higher end shows more dominance (Fig. 4). The curverepresenting season (Fig. 5), North-east monsoon was at the bottom denoting more diversity and summer at the top denoting less diversity. The season-wise similarity percentage results of species contribution of bycatch ichthyofauna within and between the seasons throughout the study are given in Tables 3 and 4 .

\subsection{MDS bubble plot and linkage tree}

The season-wise bycatch ichthyofauna abundance divulges the maximum abundance during Post-monsoon (27.83\%) followed by North-east monsoon (27.03\%), Summer (23.44\%) and the Southwest monsoon (21.70\%). The MDS bubble spot reveals an abundance of fish species and dissimilarity between the seasons that are denoted by different sizes of circle, where larger size bubbles represent maximum abundance (Fig. 6). Among the fish groups, Leiognathus equulus was the presiding fish species with supreme abundance during South-west and North-east monsoon (Fig. 7).

The 95\% confidence funnel and ellipse were raised for the average taxonomic distinctness index $(\Delta+)$ and variation in taxonomic distinctness index $\left(/ \Lambda^{+}\right)$values of all the season, which reveals that all seasons were well fit within the 95\% confidence funnel showing no crucial deviation (Figs. 8, 9 \& 10). Linkage tree was drawn to find out the variation in average rank dissimilarities between and within the various seasons by a threshold on more variables. The split $(A)$ in the schismatic clustering between 1 , 4 and 2,3 provides an optimal $\mathrm{R}$ of 1.00 with division $\mathrm{B} \%=$ 100 , presented on the y-axis. The split (A) discrete from others depends on the few markedly abundant bycatch ichthyofauna species, which are represented in Fig. 11.

\subsection{Diversity of fishes within various trophic levels (TrLs) and habi-} tat

The trophic level (TrLs) of planktivores/herbivores was within the range of 2.0-2.49 contributing two species which accounts $1 \%$, seven species were in the range of $2.5-2.99$ (primary carnivores) contributing 5\%, 41 species in the range of 3.0-3.49 (mid-level carnivores) contributes $26 \%, 77$ species were in the range 3.5-3.99 (top-level carnivores) contributing 49\% and 29 species were in the range of 4.0-4.5 (apex predators) contributing 19\%. The minimum TrLs was observed in Siganus lineatus (2.0) and Acanthurus mata (2.2), which are reef-associated fishes feeding mainly algae on the beach rock or coral reefs. Sphyraena jello Cuvier, 1829, Ichthyscopus lebeck (Bloch and Schneider, 1801) and Torpedo sinuspersici (Olfers, 1831) were top in TrLs with 4.5, 
Table 3

Seasonal similarity percentages (SIMPER) within different seasons in the species contributions of bycatch ichthyofauna for the study period.

\begin{tabular}{|c|c|c|c|c|c|}
\hline Species & $\begin{array}{l}\text { Average } \\
\text { abundance (\%) }\end{array}$ & $\begin{array}{l}\text { Average } \\
\text { similarities (\%) }\end{array}$ & $\begin{array}{l}\text { Average } \\
\text { dissimilarities (\%) }\end{array}$ & $\begin{array}{l}\text { Average } \\
\text { contribution (\%) }\end{array}$ & $\begin{array}{l}\text { Cumulative } \\
\text { contribution (\%) }\end{array}$ \\
\hline \multicolumn{6}{|c|}{ Bycatch ichthyofauna Average similarity: 82.05} \\
\hline Leiognathus equulus & 7.97 & 1.47 & 36.79 & 1.79 & 1.79 \\
\hline Equulites lineolatus & 7.92 & 1.46 & 57.82 & 1.78 & 3.58 \\
\hline Secutor insidiator & 7.89 & 1.45 & 50.78 & 1.77 & 5.34 \\
\hline Gazza achlamys & 7.74 & 1.43 & 48.22 & 1.75 & 7.09 \\
\hline Thryssa malabarica & 7.73 & 1.42 & 57.38 & 1.73 & 8.82 \\
\hline Karalla dussumieri & 7.54 & 1.4 & 41.46 & 1.7 & 10.52 \\
\hline Otolithes ruber & 7.27 & 1.33 & 28.74 & 1.62 & 12.14 \\
\hline Trypauchen vagina & 7.5 & 1.31 & 21.64 & 1.59 & 13.74 \\
\hline Nuchequula blochii & 7.02 & 1.3 & 37.89 & 1.58 & 15.32 \\
\hline Nibea maculata & 6.94 & 1.28 & 42.57 & 1.56 & 16.87 \\
\hline Grammoplites scaber & 6.61 & 1.22 & 34.9 & 1.49 & 18.36 \\
\hline Encrasicholina punctifer & 6.86 & 1.22 & 12.89 & 1.48 & 19.84 \\
\hline Trachinocephalus myops & 6.86 & 1.2 & 8.98 & 1.46 & 21.31 \\
\hline Johnius dussumieri & 6.38 & 1.19 & 34.43 & 1.45 & 22.76 \\
\hline Uroconger lepturus & 6.47 & 1.18 & 55.79 & 1.44 & 24.19 \\
\hline Parachaeturichthys polynema & 6.35 & 1.17 & 23.57 & 1.43 & 25.63 \\
\hline Stolephorus commersonnii & 6.69 & 1.17 & 11.06 & 1.43 & 27.05 \\
\hline Grammoplites suppositus & 6.29 & 1.16 & 41 & 1.41 & 28.47 \\
\hline Synchiropus lineolatus & 6.16 & 1.14 & 25.61 & 1.38 & 29.85 \\
\hline Stolephorus indicus & 6.4 & 1.13 & 12.12 & 1.38 & 31.23 \\
\hline Saurida tumbil & 6.66 & 1.13 & 7.19 & 1.38 & 32.61 \\
\hline Narcine brunnea & 6.13 & 1.13 & 52.32 & 1.37 & 33.98 \\
\hline Parapercis alboguttata & 6.07 & 1.1 & 34.38 & 1.34 & 35.33 \\
\hline Ostorhinchus fasciatus & 5.99 & 1.1 & 55.73 & 1.34 & 36.67 \\
\hline Selaroides leptolepis & 6.13 & 1.1 & 20.58 & 1.34 & 38.01 \\
\hline Gymnothorax reticularis & 6.06 & 1.09 & 19.69 & 1.33 & 39.34 \\
\hline Lagocephalus suezensis & 6.82 & 1.09 & 3.61 & 1.33 & 40.67 \\
\hline Torpedo sinuspersici & 5.98 & 1.08 & 65.83 & 1.32 & 41.99 \\
\hline Jaydia ellioti & 5.92 & 1.08 & 32.06 & 1.32 & 43.31 \\
\hline Upeneus vittatus & 5.9 & 1.08 & 31.38 & 1.31 & 44.62 \\
\hline Narke dipterygia & 5.95 & 1.07 & 52.17 & 1.31 & 45.93 \\
\hline Terapon jarbua & 6.01 & 1.05 & 10.64 & 1.28 & 47.21 \\
\hline Trichiurus lepturus & 5.89 & 1.05 & 15.25 & 1.28 & 48.49 \\
\hline Zebrias quagga & 5.71 & 1.02 & 31.15 & 1.25 & 49.74 \\
\hline Gymnothorax prolatus & 5.67 & 1.02 & 30.29 & 1.24 & 50.98 \\
\hline
\end{tabular}

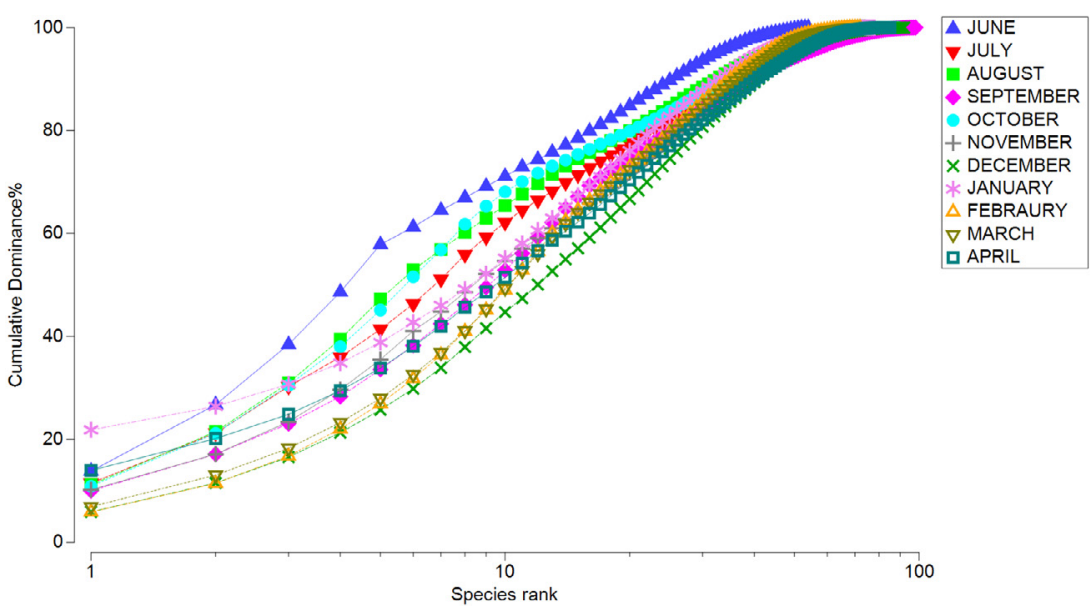

Fig. 4. K-Dominance plot among different months for bycatch of trawl net fishery of Chennai coast.

which feeds mainly on bony fishes, squids and benthic invertebrates. Shannon-Wiener species diversity ( $\left.\mathrm{H}^{\prime}\right)$ was estimated for various TrLs and found to be within the range of $0.02-3.12$ (minimum Trls 2.0-2.49; maximum TrLs 3.5-3.99). Margalef species richness (d) were in the range of 0.17-6.49 (minimum TrLs 2.02.49; maximum TrLs 3.5-3.99). Pielous's evenness (J') ranges from 0.03-0.73 (minimum TrLs 2.0-2.49; maximum TrLs 4.0-4.5) (Table 5). The dendrogram revealed that maximum similarities (77\%) were shown in between the trophic level TrLs 3.0-3.49 (mid-level carnivores) and 4.0-4.5 (apex-predator) in species composition than other $\operatorname{TrL}$ (Fig. 3). From the anaysis of the trophic level values of 156 fish species of the trawl bycatches from this region was found to be predominant by top-level carnivores group with high species diversity ( $\left.\mathrm{H}^{\prime}\right)$ with the value of 3.12 for 77 species. Among the 156 bycatch fish species from the region of Chennai coast, 72 species were demersal (46\%), 70 species were reef-associated (45\%), eight species were benthopelagic (5\%) five species were pelagic (3\%) and one bathydemersal species (1\%). 


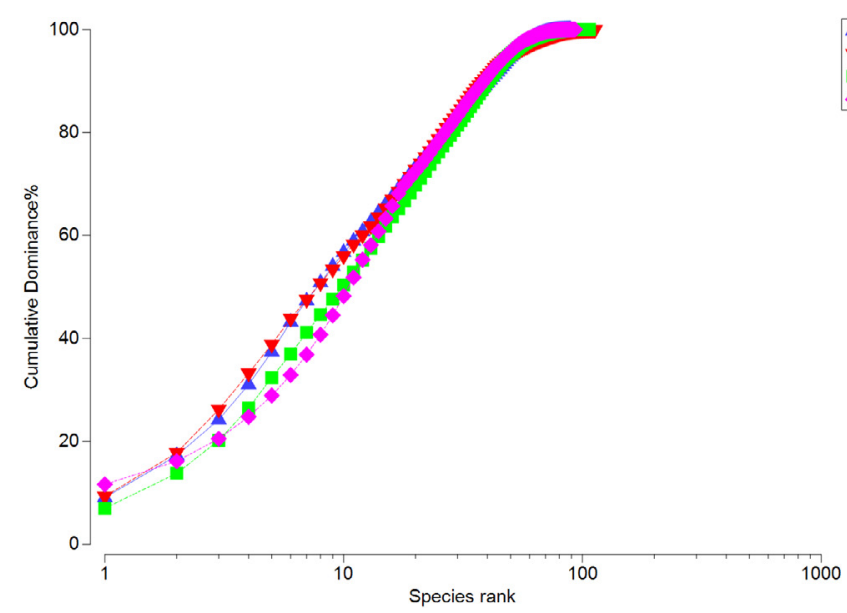

Fig. 5. K-Dominance plot among different seasons for bycatch of trawl net fishery of Chennai coast.

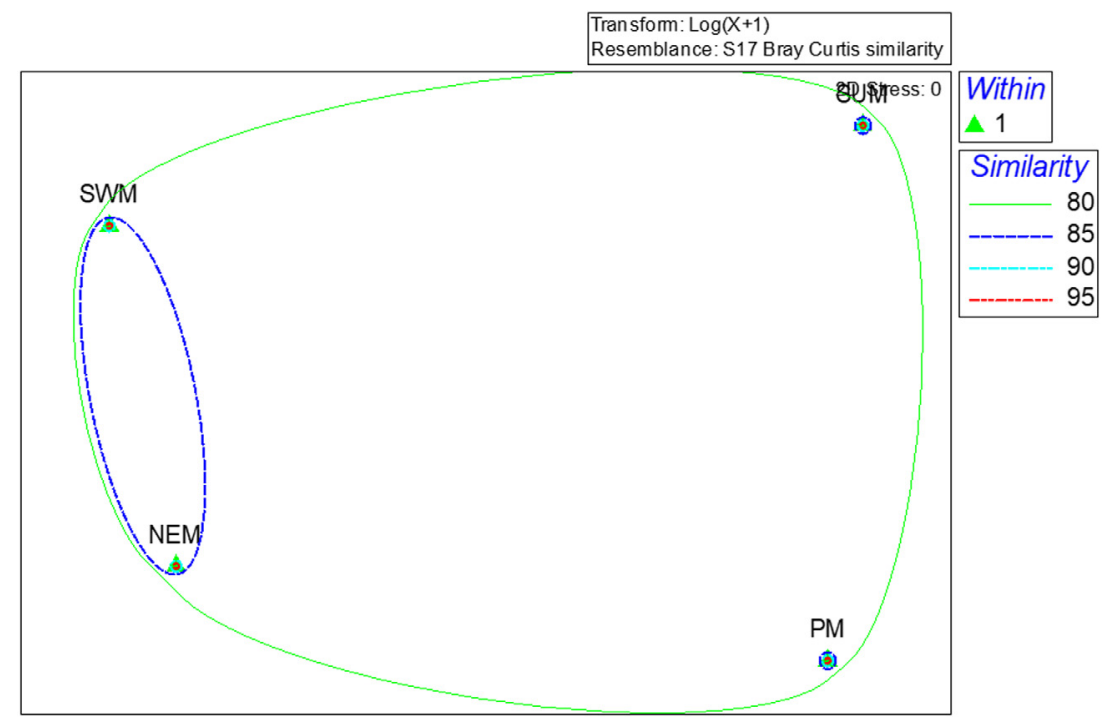

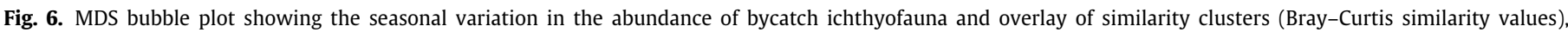

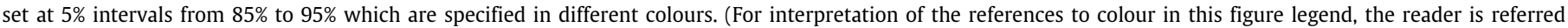
to the web version of this article.)

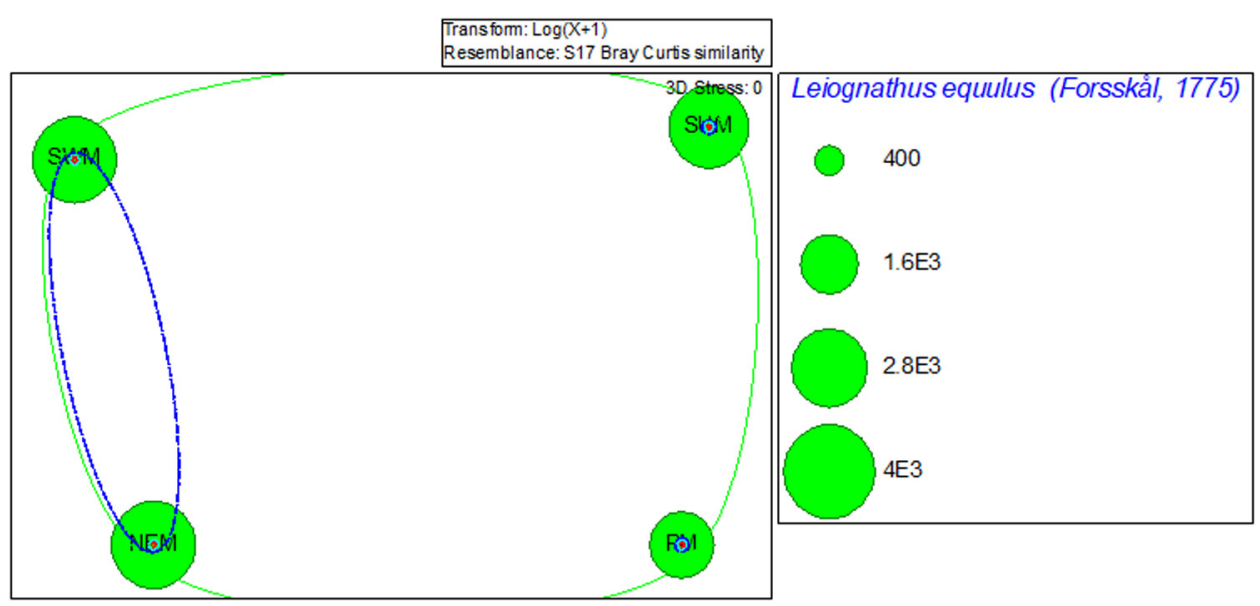

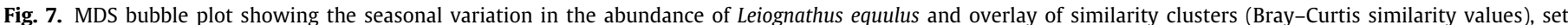

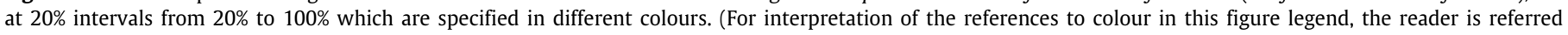
to the web version of this article.) 
Table 4

Seasonal similarity percentages (SIMPER) between different seasons in the species contributions of bycatch ichthyofauna for the study period.

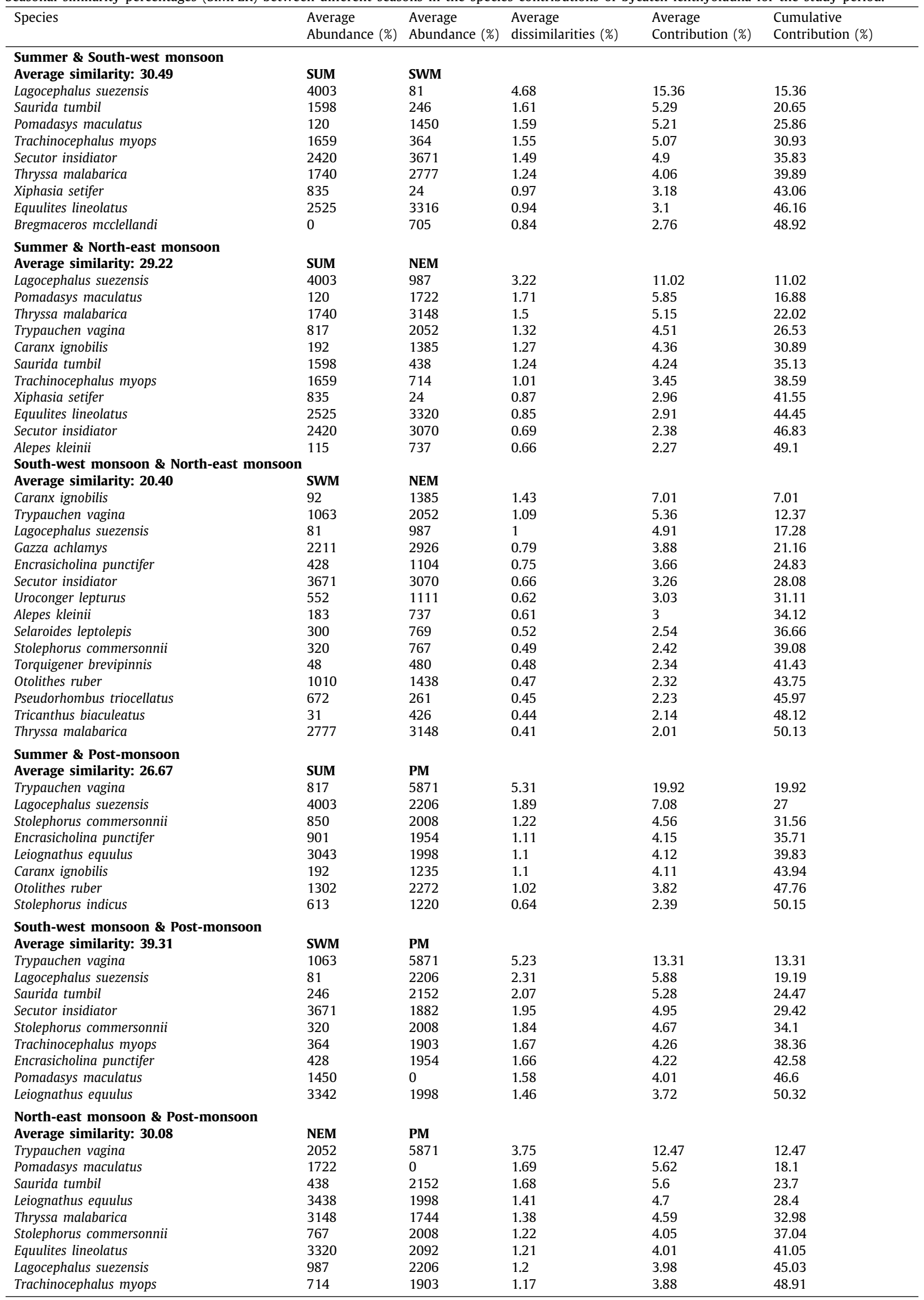




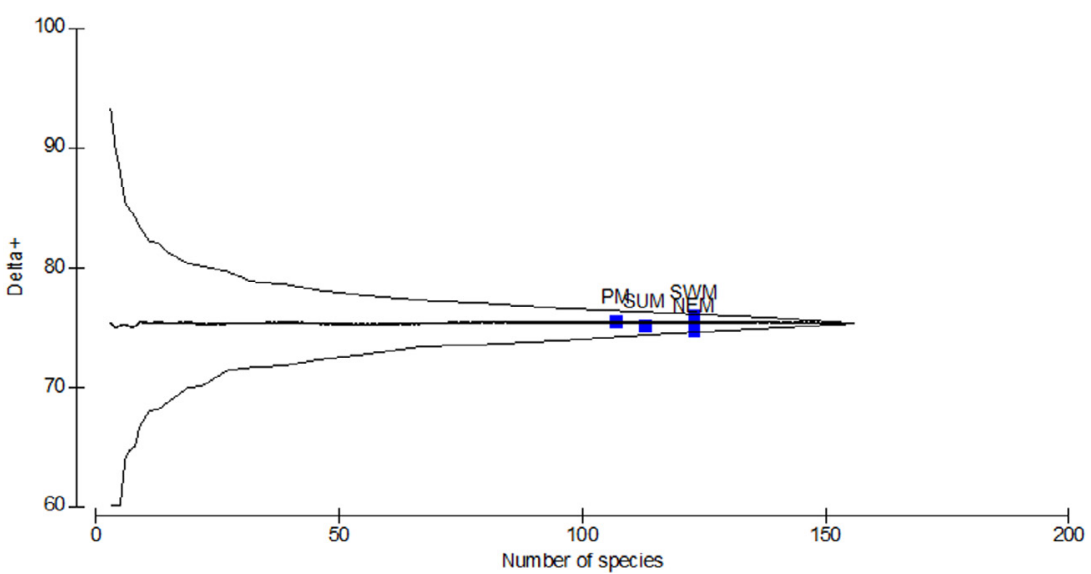

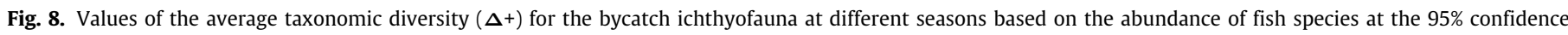
funnel and deviation from the normal distribution from the mean delta + as dotted line.

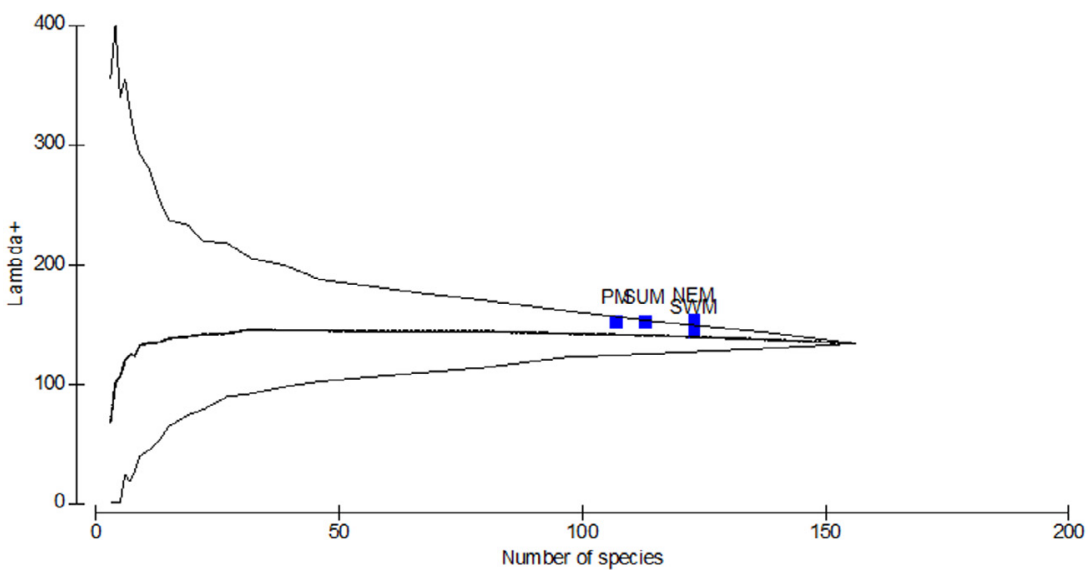

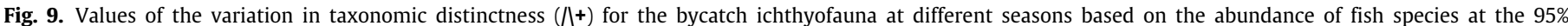
confidence funnel and deviation from the normal distribution from the mean lambda+ as dotted line.

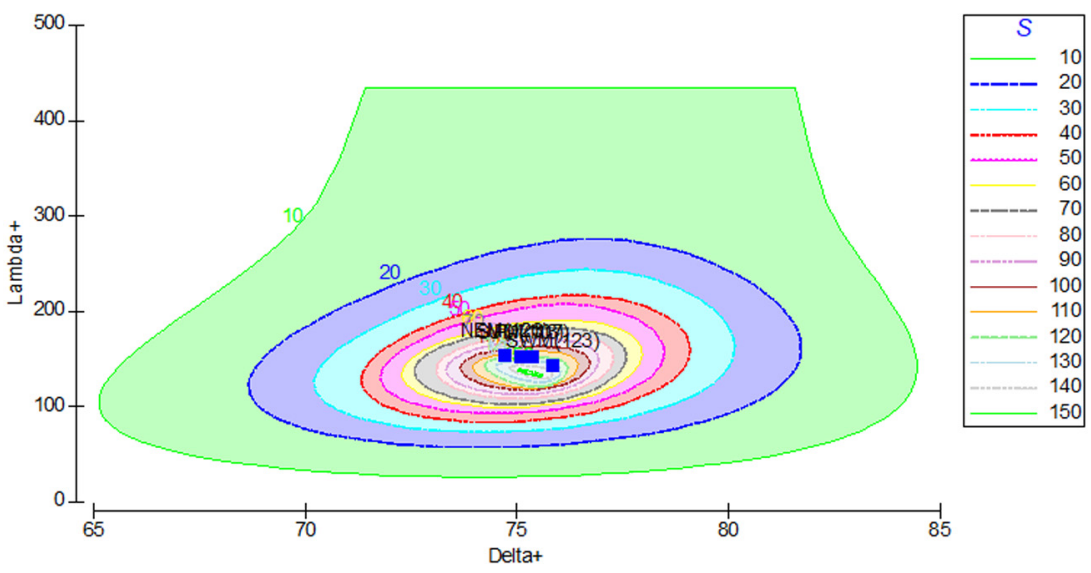

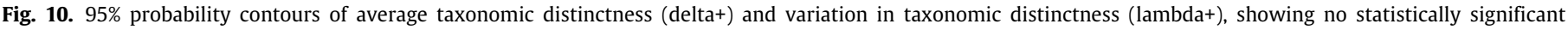
deviation in bycatch ichthyofauna diversity between seasons.

\section{Discussion}

\subsection{Bycatch studies in Indian coast}

The initial survey on assessment of bycatch in Indian marine fisheries carried out in 1979 revealed that 79\% of non-target fishes were mostly landed by shrimp trawl (George et al., 1981). During $1980-82$, $85 \%$ of bycatches were landed from shrimp trawlers operating along the Karnataka coast (Sukumaran et al., 1982). Menon (1996) estimated bycatch landing off the southern coast of India during 1985-90, which revealed 1.3 lakh tonnes of non-edible benthic organisms landed by trawlers along the coast of Karnataka, Kerala and Tamil Nadu. A huge quantity of 2.62 and 2.25 lakh tonnes of marine organisms were discarded during 2000-01 and 2001-02 respectively along the Kerala coast, which included 103 fish species returned back to sea, which 

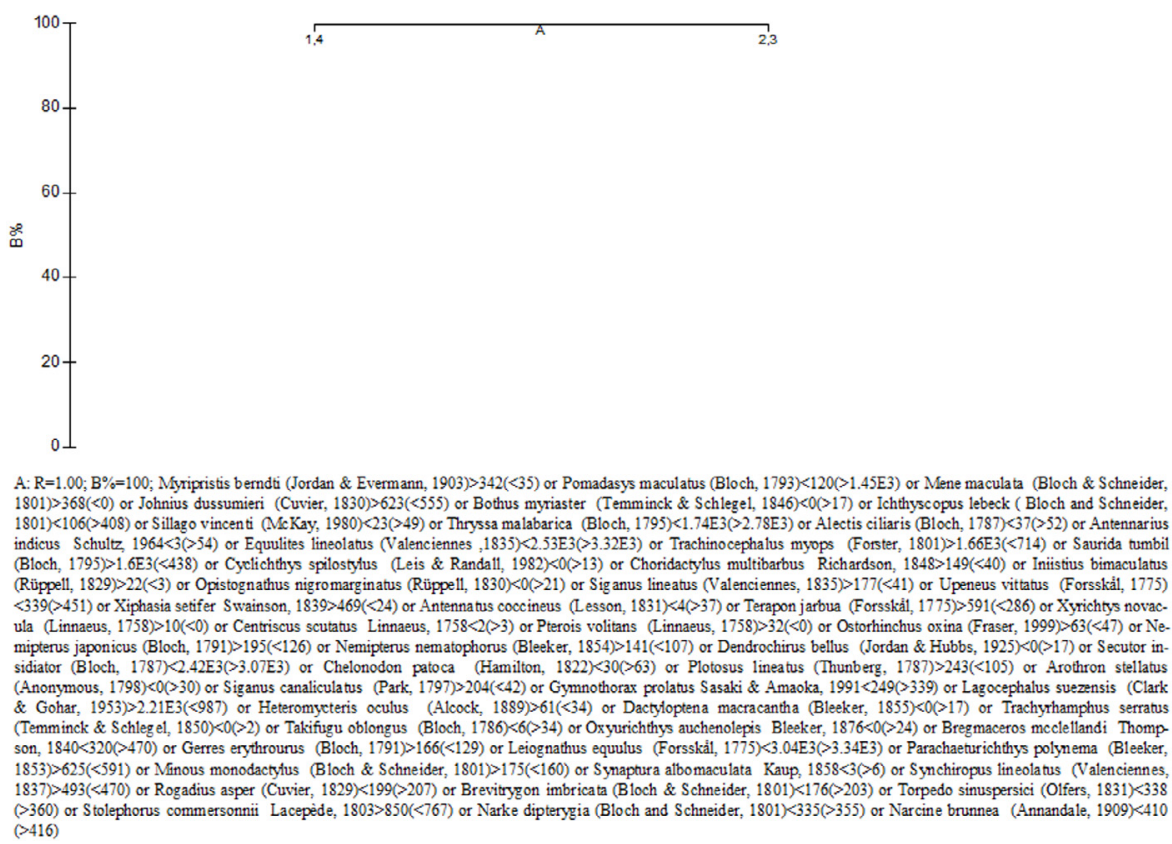

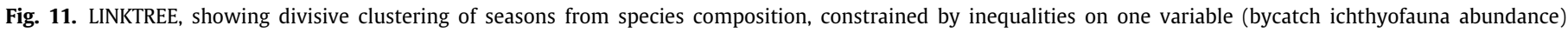
with ANOSIM R value and B \% for each split.

Table 5

Trophic Level (Trls) diversity indices of fish fauna in bycatch of trawl net fishery of Chennai coast.

\begin{tabular}{lllll}
\hline TrLs & S & $d$ & J' & $\mathrm{H}^{\prime}$ \\
\hline $2.0-2.49$ & 2 & 0.1679 & 0.0260 & 0.0180 \\
$2.5-2.99$ & 7 & 0.6197 & 0.4653 & 0.9054 \\
$3.0-3.49$ & 44 & 3.9701 & 0.6702 & 2.5361 \\
$3.5-3.99$ & 74 & 6.4933 & 0.7243 & 3.1174 \\
$4.0-4.5$ & 29 & 2.7712 & 0.7274 & 2.4492 \\
\hline
\end{tabular}

S- Number of species; $d$-Margalef's species richness; J'- Pielous's evenness; H'Shannon-Wiener species diversity. 2.0-2.49- herbivores/planktivores; 2.5-2.99primary carnivores; 3.0-3.49- midlevel carnivores; 3.5-3.99- top-level carnivores 4.0-4.5- apex predator.

were all exploited as bycatch in bottom trawlers (Kurup et al., 2003). Bycatch fish fauna associated with shrimp trawlers along the coast of Cuddalore and Parangipettai coast was studied by Murugesan et al. (2013) and Sambandamoorthy et al. (2015). Dineshbabu et al. (2013) estimated the changing trend in bycatch landing of trawl fisheries of India between 2008 and 2011. Velip and Rivonker (2015) estimated composition and trends in trawl bycatches on tropical fishing ground on Goa. Samanta et al. (2018) estimated the relationship between lunar cycle and shrimp trawl bycatches of Mumbai coast. Ranjith et al. (2018) estimated the diversity and biomass of plant biota, which are exploited as bycatch from trawlers along the Thoothukudi coast. Ramkumar et al. (2019) performed qualitative and quantitative analysis of non-targets, diversity and species composition in trawl bycatches from the northern Maharashtra coast. Bijukumar and Deepthi (2009) evaluated the mean trophic index of fish fauna of about 3.12 from trawl bycatches of Kerala coast. However, the present study differs from the earlier studies which finds the mean trophic index of fish fauna where increased diversity and temporal variations of bycatch ichthyofauna are associated with the trophic level of the Chennai coast.

\subsection{Diversity of ichthyofauna associated with trawl bycatches}

Pillai et al. (2014) studied the diversity of crustacean bycatch of trawlers along the Chennai coast during 2005 to 2009 recorded
64 species whereas in the present study records 156 species of ichthyofauna from the trawl bycatches of Chennai coast during 2018-19. Dineshbabu et al. (2013) studied the diversity of fish species in the bycatch from major landing centres along the west coast of India and revealed 41 fish species from Veraval, 51 species from Mumbai; 57 species from Karwar and 95 species from Mangalore coast whereas the present study recorded higher fish faunal diversity. However, Visakhapatnam coast recorded 228 bycatch species (Sujatha, 1996) which is much higher when compared to the present study. Zacharia et al. (2006) recorded 53 fish species from the discards of trawlers operating along the coast of Karnataka whereas Dineshbabu et al. (2010) collected a maximum of 116 species of finfishes from the trawl bycatch along Karnataka coast. The study reveals variation in the species diversity in terms of the number of bycatch species depending upon various factors viz., operating fishing grounds, fishing gear used and seasons. Sirajudheen and Biju Kumar (2014) recorded 14 orders, 67 families of 138 species of ornamental fishes belonging to 108 genera. Among this, the order Perciformes contributed the maximum number of species with 77 species, followed by Tetraodontiformes (21 species) and Scorpaeniformes (17 species). Scorpaenidae ( 9 species) was a species-rich family, followed by Serranidae (7 species) and Tetraodontidae (7 species) from trawl by-catch of the Neendakara fishing harbour of Kerala. On par with that the present study also recorded lower marine ornamental fish diversity i.e., 34 species belonging to 14 families. Sambandamoorthy et al. (2015) reported 123 species of finfishes from trawl bycatches along the coast of Cuddalore and Parangipettai however, the present study along the Chennai coast has more fish species diversity.

Mahesh et al. (2019) noticed that $47.5 \%$ of trawl bycatch consisted of juveniles of commercially important finfishes of Karnataka coast. Bijukumar and Deepthi (2009) noted that the length class of most commercial ichthyofauna in all the trophic level associated with trawl bycatches were less than $15 \mathrm{~cm}$ and in the present study most of the fish species were noticed with size ranges less than $12 \mathrm{~cm}$ which clearly shows that bycatch landings of Chennai coast consists of more juvenile fish groups. In addition, cod end mesh size (CEMS) plays a vital role in harvest of varied 
size ranges, though CEMS with a maximum of $47 \mathrm{~mm}$ to facilitate the easier escaping of juveniles to ensure sustained production of benthic resources (Rajeswari et al., 2010).

\subsection{Diversity indices}

Pillai et al. (2014) estimated the seasonal H' value in the range of 4.76 (Summer 2006)-5.59 (South-west monsoon 2008) with 32-52 crustacean species in the bycatch of trawl fishery along the Chennai coast. Similar observations were made by Sambandamoorthy et al. (2015) where the H' values were within the range of $0.84-4.52$ with the presence of 8 to 49 fish species from trawl bycatch of Cuddalore and Parangipettai coasts. Murugesan et al. (2013) estimated the H' values ranging between 1.223.16 from trawl bycatch of the Parangipettai coast and 2.25-3.52 with the presence of 41 to 60 fish species from Cuddalore coast. Similarly, in the present study, monthly species diversity $\left(\mathrm{H}^{\prime}\right)$ values were in the range of 4.53 (June)-5.63 (December) with the presence of 59-105 fish species. The Shannon-Weiner diversity index calculated in the present study revealed that bycatch of trawl net fishery of the Chennai coast is more diverse in nature with respect to finfish species. The lower H' value recorded during June might be due to a trawl ban along the Tamil Nadu coast, which was imposed from April 15th to June 15th. The higher value recorded during December may be due to the influence of the North-east monsoon, which increased freshwater inflow into coastal waters through coastal or river rainfall drainages resulting in increased primary production which would attract the more fish community, resulting in high diversity.

The seasonal Margalef species richness (d') calculated in the present study varied from 9.77 during Post-monsoon to 11.51 during South-west monsoon with the presence of 107-123 fish species. Likewise, Pillai et al. (2014) estimated the d' values ranged from 7.18 (North-east monsoon) to 10.71 (South-west monsoon) in 2005. The high range (d') value in this present study might be due to more number of species recorded from the bycatch of trawl net fishery of the Chennai coast compared to earlier studies of Pillai et al. (2014). Likewise, Sambandamoorthy et al. (2015) estimated the d' values that fell within the range of 0.34-4.84 from trawl bycatch of Cuddalore and Parangipettai coasts. Murugesan et al. (2013) estimated the d' values within the range of $1.30-2.76$ from trawl bycatch of the Parangipettai coast and 1.72-2.83 from trawl bycatch of Cuddalore coast. The monthly Margalef species richness calculated in the present study varied from 6.33 during June to 11.65 during September with the presence of 59 to 112 fish species. On par with that, Pillai et al. (2014) also estimated the monthly Margalef species richness ranging between 2.4 during April 2006 and 10.28 during July 2008. The relative abundance for species occurring during various seasons clearly showed maximum abundance during South-west monsoon which could be attributed to more productivity due to rainfall, whereas summer season encountered minimum relative abundance due to the less fishing activity by dint of 61 days common fishing ban along the east coast of India. Thus, it is evident from the present study that the higher d' values were attributed to more numbers of species recorded in line with earlier similar studies.

Seasonal Pielou's evenness (J') calculated in the present study was in the range of $0.77-0.81$ (123 to 107 species). Meanwhile, Pillai et al. (2014) estimated the J' value in the range of 0.940.98 (39 to 52 species) for crustacean bycatch from trawl fishery along the north Tamil Nadu. Monthly Pielou's evenness (J') calculated in the present study was in the range of $0.75-0.84$ (104 to 88 species). Similar observations were made by Murugesan et al. (2013) with J' values within the range of 0.71-0.92 from Parangipettai coast and 0.54-0.89 from Cuddalore coast. The species evenness was lower at South-West Monsoon, which may be due to the high number of species or dominance of individual species as revealed by Clarke and Warwick (2001).

Among the four seasons, the highest taxonomic diversity $(\Delta)$ value of the present study was found in Post-monsoon (86.08) and the lowest in South-west monsoon (81.85). Among the months, the highest taxonomic diversity $(\Delta)$ value was found in December (87.68) with the presence of 105 species and the lowest in June (76.71) with the presence of 59 species. Meanwhile, Murugesan et al. (2013) estimated the taxonomic diversity ( $\Delta$ ) falling within the range of 43.25 (October) to 52.60 (November) for Parangipettai coast and 42.08 (March) to 52.31 (December) for Cuddalore coast. In the present study, the lowest value obtained in June shows that the diversity was poor in June when compared to other months, which might be due to the commencement of the target fishing operation soon after the trawl ban period. The average taxonomic distinctness $(\Delta+)$ value for seasons ranged from 91.82 North-east monsoon to 93.24 (South-west monsoon). The average taxonomic distinctness $\left(\Delta^{+}\right)$value for months ranged from 89.51 (June) to 92.77 (September). Thus, the average taxonomic distinctness $(\Delta+)$ shows a positive correlation with species richness with more species in September and a minimum in June. Murugesan et al. (2013) made a similar observation for reef fish communities from the Parangipettai and Cuddalore coast.

The samples from biodiversity-rich areas have more stability and less variation in taxonomic distinctness which was observed in September 168.67 with the presence of 112 species and more variation in taxonomic distinctness was in June 207.74 with 59 species. This hypothesis was specified by Clarke and Warwick (2001). In this present study, monthly total phylogenetic diversity (sPhi+) ranged from 3750 (June) to 6500 (September) and seasonwise total phylogenetic diversity (sPhi+) ranged from 6525 (Postmonsoon) to 7075 (South-west and North-east monsoon). Similarly, Murugesan et al. (2013) reported total phylogenetic diversity (sPhi+) for trawl bycatch from the coasts of Parangipettai and Cuddalore. The values varied from 466.66 (April) to 966.66 (August) for the Parangipettai coast and 550 (February) to 883.33 (September) for the Cuddalore coast. Thus, the present study on trawl bycatch of the Chennai coast showed higher diversity than the study of Murugesan et al. (2013), which dealt with trawl bycatch of the coasts of Parangipettai and Cuddalore. The result of the K-dominance curve plotted with month-wise and seasonwise data revealing high diversity and more species richness was observed in December and North-east Monsoon than the other months and seasons which may be attributed to the maximum rainfall experienced in the North-east monsoon, resulting in increased primary production due to enormous input of freshwater into coastal waters through river or coastal drainages (Hoguane et al., 2012). The present comparative diversity analysis reveals that there was a great temporal variation in species diversity of trawl bycatches of Chennai coast when compared to the coast of southern India.

\subsection{Simper, taxtest and linktree}

In the present study, average seasonal similarity percentages (SIMPER) within different seasons in the species contributions of bycatch ichthyofauna were $82.05 \%$. Similarly, Ramkumar et al. (2019) found out $72.10 \%$ of seasonal similarities within the different seasons during 2013-14 for finfish bycatch along the northern Maharashtra coast. Ranjith et al. (2018) estimated the SIMPER for marine biota, which was exploited as bycatch along the Thoothukudi coast and revealed $34.68 \%$ similarity for the seaweeds and $49.38 \%$ for seagrasses. Between the different seasons, the maximum similarity was seen between southwest monsoon and post-monsoon (39.31\%). Ranjith et al. (2018) showed the 
maximum similarity for seaweeds (53.31\%) during summer and pre-monsoon and for seagrasses, maximum similarity (75.86\%) during post-monsoon and monsoon. In this study, Leiognathus equulus contributed $7.97 \%$ (with $1.47 \%$ average similarity) to the total similarity of trawl bycatches of Chennai coast. Meanwhile, Varghese et al. (2017) reported that Siganus caniculatus contributed a maximum of $23.82 \%$ similarities to total similarity of reef fishes between the 2006 and 2012 along the southeast coast of India. The $95 \%$ confidence funnel of average taxonomic distinctness index $\left(\Delta^{+}\right)$and variation in taxonomic distinctness index $(/+)$ values, showed no significant deviation in bycatch fish diversity between the seasons. Varghese et al. (2017) stated that 95\% probability contours showed significant deviation in reef fish diversity at Keelakarai, Gulf of Mannar between 2006 and 2012 . In this study, the $95 \%$ probability contours of average taxonomic distinctness index $(\Delta+)$ and variation in taxonomic distinctness index $\left(\Lambda^{+}\right)$values were well within the funnel showing no significant deviation. Moreover, the species variation between the seasons is less due to high values in bycatch ichthyofauna diversity.

\subsection{Diversity of fish within various trophic level and habitat}

In the present study, demersal habitat fishes were observed as the dominant group in the trawl bycatches of Chennai Coast. Similarly, along the west coast of India, Bijukumar and Deepthi (2009) reported that trawl bycatches were dominant with reefassociated and demersal fish groups. Likewise, along the coast of Cuddalore and Parangipettai situated along the east coast of India, Sambandamoorthy et al. (2015) stated that trawl bycatch was with the dominance of demersal and reef-associated fish groups. Observation of demersal habitat fish species as a dominance group in the present study is further owing to the bottom trawlers standing as major fishing gear in the region. Trophic level interactions play a vital role in structural maintenance and ecosystem functioning (Longo et al., 2014) and also provide a better representation of habitats occupied by the species (Herbert et al., 1999). Furthermore, trophic level estimations show better prophecy for species ecological performance, habitat selection, riposte to inconvenient environmental conditions (Violle et al., 2007). In the present study, the significant differences in trophic association and assemblages of fishes in respective habitats were observed during the various seasons to figure out the consequential variations in bycatch fish diversity. Trophic level results as opined by Bijukumar and Deepthi (2009) and Sambandamoorthy et al. (2015), revealed that the trophic level of fish bycatch from trawl nets dominated at the TrLs of 3.5-3.99. Bijukumar and Deepthi (2009) epitomized that lower trophic level species was Acanthurus bleekeri which is in conformity to the present study by the presence of species belonging to similar generus i.e., Acanthurus mata. Over-representation and abundance of toplevel carnivores (TrLs 3.5-3.99) in the bycatch of trawlers may be the reason for annihilation of the primary and mid-level predators from the bycatch harvesting ecosystem, which indirectly affects diversity of marine landings. In the same line, dominance of top-level carnivores observed in the present study, might be a major cause for alteration of trophic levels in ichthyofaunal bycatch that may affect the diversity of the Chennai coast.

\section{Conclusion}

The present study gives the baseline information on the ichthyofaunal diversity from trawl bycatch. The study reveals three new records to Indian waters which indicates that this region is a potential ground for exploring more Ichthyofaunal species diversity. However, the observation of juveniles of commercially important fish from the bycatch composition highlights the juvenile fishing which can create a major impact on the marine fisheries resources. This in turn affects the species composition, trophic level and the stock along the region. The impact on the species may affect trophic levels leading to destabilization of benthic communities. Hence, there is a need to take necessary steps to implement existing conservation and management measures effectively. In addition, community participation by involvement of various stakeholders and voluntary implementation management measures imposed by the government would be more effective in minimizing the bycatch thereby safeguarding our marine fishery resources for future generation.

\section{CRediT authorship contribution statement}

Paramasivam Kodeeswaran: Conceptualization, Methodology, Software, Validation, Investigation, Data curation, Writing original draft, Writing - review \& editing, Visualization. Natarajan Jayakumar: Conceptualization, Methodology, Formal analysis, Investigation, Data curation, Writing - review \& editing, Visualization, Supervision. Lakshmanan Ranjith: Methodology, Software, Formal analysis, Writing - review \& editing, Visualization, Supervision.

\section{Declaration of competing interest}

The authors declare that they have no known competing financial interests or personal relationships that could have appeared to influence the work reported in this paper.

\section{Acknowledgements}

The authors thank Dr. B. Ahilan, Dean, Dr. M.G.R. Fisheries College and Research Institute, Ponneri for providing necessary facilities. The first author thanks Mr. J. Praveenraj, Scientist, ICARCentral Island Agricultural Research Institute, Port Blair, Andaman and Nicobar Islands, for his massive help during research work more specifically on fish identification. The first author also thanks Mr. K. Karuppasamy, Teaching Assistant, College of Fisheries Nautical Technology, Thoothukudi and Mr. N. Moulitharan (PG Scholar) for helping in field sampling and support during the entire study.

\section{References}

Bijukumar, A., Deepthi, G.R., 2009. Mean trophic index of fish fauna associated with trawl bycatch of Kerala, southwest coast of India. J. Mar. Biol. Assoc. India 51 (2), 145-154.

Christensen, V., Pauly, D., 1992. ECOPATH II-a software for balancing steadystate ecosystem models and calculating network characteristics. Ecol. Mod. 61 (34), 169-185.

Clarke, K.R., 1993. Non-parametric multivariate analyses of changes in community structure. Aust. J. Ecol. 18, 117-143.

Clarke, K.R., Gorley, R.N., 2015. Getting Started with PRIMER v7. PRIMER-E: Plymouth. Plymouth Marine Laboratory.

Clarke, K.R., Somerfield, P.J., Gorley, R.N., 2008. Testing of null hypotheses in exploratory community analyses: similarity profiles and biota-environment linkage. J. Exp. Mar. Biol. Ecol. 366, 56-69.

Clarke, K.R., Warwick, R.M., 1998. A taxonomic distinctness index and its statistical properties. J. App. Eco. 35 (4), 523-531.

Clarke, K.R., Warwick, R.M., 2001. A further biodiversity index applicable to species lists: variation in taxonomic distinctness. Mar. Eco. Prog. Ser. 216, $265-278$.

Day, F., 1878. The Fishes of India; Being a Natural History of the Fishes Known to Inhabit the Seas and Fresh Waters of India, Burma, and Ceylon. Bernard Quaritch, London, http://dx.doi.org/10.5962/bhl.title.55567, Part IV, i-xx + 553-778, [ref. 1080]. 
Dineshbabu, A.P., Radhakrishnan, E.V., Thomas, S., Maheswarudu, G., Manojkumar, P.P., Kizhakudan, S.J., Pillai, S.L., Chakraborty, R.D., Josileen, J., Sarada, P.T., Sawant, P.B., 2013. Appraisal of trawl fisheries of India with special reference on the changing trends in bycatch utilization. J. Mar. Biol. Assoc. India 55 (2), 69-78.

Dineshbabu, A.P., Sujitha, T., Radhakrishnan, E.V., 2010. Bycatch from trawlers with special reference to its impact on commercial fishery, off Mangalore. In: Coastal Fishery Resources of India - Conservation and Sustainable Utilisation. Central Institute of Fisheries Technology, Kochi, pp. 327-334.

Eastwood, P.D., Mills, C.M., Aldridge, J.N., Houghton, C.A., Rogers, S.I., 2007. Human activities in UK offshore waters: an assessment of direct, physical pressure on the seabed. ICES J. Mar. Sci. 64 (3), 453-463.

FAO, 2020. The state of world fisheries and aquaculture 2020. In: Sustainability in Action. Rome. p. 244, http://dx.doi.org/10.4060/ca9229en.

Fischer, W., Bianchi, G., 1984. FAO species identification sheets for fishery purposes. Western Indian Ocean (Fishing Area 51). v. 1: Introductory material. Bony fishes, families: Acanthuridae to Clupeidae.-v. 2: Bony fishes, families: Congiopodidae to Lophotidae.-v. 3:. families: Lutjanidae to Scaridae.-v. 4: families: Scatophagidae to Trichiuridae.-v. 5: Bony fishes, families: Triglidae to Zeidae. Chimaeras. Sharks. Lobsters. Shrimps and prawns. Sea turtles. v. 6: Alphabetical index of scientific names and vernacular names.

Fischer, W., Whitehead, P.J.P., 1974. FAO Species Identification Sheets for Fishery Purposes: Eastern Indian Ocean (Fishing Area 57) and Western Central Pacific (Fishing Area 71), Vol. 2. Food and Agriculture Organization of the United Nations.

Foden, J., Rogers, S.I., Jones, A.P., 2011. Human pressures on UK seabed habitats: a cumulative impact assessment. Mar. Eco. Prog. Ser. 428, 33-47.

Froese, R., Pauly, D. (Eds.), 2018. FishBase. World Wide Web Electronic Publication, https://www.fishbase.org, [version 06/2018].

George, M.J., Suseelan, C., Balan, K., 1981. By-catch of shrimp fisheries in India. Mar. Fish. Inf. Serv. Tech. Ext. Ser. 28, 3-13.

Gilman, E., Huntington, T., Kennelly, S.J., Suuronen, P., Chaloupka, M., Medley, P., 2019. In: Pérez Roda, M.A. (Ed.), A Third Assessment of Global Marine Fisheries Discards. In: FAO Fisheries and Aquaculture Technical Paper, vol. 633, FAO, Rome, p. 78.

Gon, O., Randall, J.E., 2003. A review of the Cardinalfishes (Perciformes: Apogonidae) of the red sea. Smithiana 1, 1-46, 10 figs., 3 tabs., 6 pls.

Herbert, D.A., Rastetter, E.B., Shaver, G.R., Ågren, G.I., 1999. Effects of plant growth characteristics on biogeochemistry and community composition in a changing climate. Ecosystems 2, 367-382. http://dx.doi.org/10.1007/ s100219900086.

Hoguane, A.M., da Lucia Cuamba, E., Gammelsrød, T., 2012. Influence of rainfall on tropical coastal artisanal fisheries a case study of Northern Mozambique. J. Integr. Coast. Zone Manage. 12 (4), 477-482.

Holthus, P.F., Maragos, J.E., 1995. Marine ecosystem classification for the tropical island Pacific. In: Marine and Coastal Biodiversity in the Tropical Island Pacific Region, Vol. 1. pp. 239-278.

Horton, T., Kroh, A., Ahyong, S., Bailly, N., Boyko, C.B., Brandão, S.N., Zhao, Z., 2018. World Register of Marine Species. WoRMS, WoRMS Editorial Board.

Kaiser, M.J., Collie, J.S., Hall, S.J., Jennings, S., Poiner, I.R., 2002. Modification of marine habitats by trawling activities: prognosis and solutions. Fish Fish. 3 (2), 114-136.

Kodeeswaran, P., Praveenraj, J., Jayakumar, N., 2020c. New record of Bearded ghoul, Inimicus didactylus (Pallas, 1769) (Scorpaeniformes: Synanceiidae) from the southeast coast of India. Thalassas http://dx.doi.org/10.1007/ s41208-020-00228-6.

Kodeeswaran, P., Praveenraj, J., Jayakumar, N., Abarna, K.M., Moulitharan, N., Mishra, S., 2020b. First record of a Shrimpgoby, Myersina yangii (Actinopterygii: Gobiiformes: Gobiidae), from Indian waters. Act. Ich. Et. Pisc. 50 (2), 219-222. http://dx.doi.org/10.3750/AIEP/02833.

Kodeeswaran, P., Praveenraj, J., Jayakumar, N., Mishra, S.S., 2020a. First report of Pseudorhombus diplospilus (Pleuronectiformes: Paralichthyidae) from east coast of India. Rec. Zoo. Sur. Ind 120 (1), 85-88. http://dx.doi.org/10.26515/ rzsi/v120/i1/2020/144892.

Kurup, B.M., Premlal, P., Thomas, J.V., Anand, Vijay, 2003. Bottom trawl discards along kerala coast: A case study. J. Mar. Biol. Assoc. India 45, 99-107.

Longo, G.O., Ferreira, C.E.L., Floeter, S.R., 2014. Herbivory drives large scale spatial variation in reef fish trophic interactions. Ecol. Evol. 4, 4553-4566. http://dx.doi.org/10.1002/ece3.1310.

Luther, G., Appanasastry, Y., 1993. Occurrence of Spawners, Juveniles and Young Fish in Relation to the Fishery Seasons of Some Major Fishery Resources of India-a Preliminary Study. In: Mar. Fish. Inf. Ser. Tech. Ext. Ser., vol. 122, pp. 1-8.

Mahesh, V., Dineshbabu, A.P., Naik, A.S., Anjanayappa, H.N., Vijaykumar, M.E., Khavi, M., 2019. Characterization of low value bycatch in trawl fisheries off karnataka coast, India and its impact on juveniles of commercially important fish species. Ind. J. Geo. Mar. Sci. 48 (11), 1733-1742.
Mallela, J., Roberts, C., Harrod, C., Goldspink, C.R., 2007. Distributional patterns and community structure of caribbean coral reef fishes within a river-impacted bay. J. Fish Biol. 70, 523-537.

Menon, N.G., 1996. Impact of bottom trawling on exploited resources. In: Menon, N.G., Pillai, C.S. (Eds.), Marine Biodiversity, Conservation and Management. Central Marine Fisheries Research Institute, Cochin, pp. 97-102.

Menon, N.G., Nammalwar, P., Zachariah, P.U., Jagadis, I., 2000. Investigations on the Impact of Coastal Bottom Trawling on Demersal Fishes and Macrobenthos. Annual Report 1999-2000, Central Marine Fisheries Research Institute, Cochin, pp. 55-57.

Murugesan, P., Silambarasan, A., Purusothaman, S., Muthuvelu, S., Anantharaman, T., 2013. Diversity of invertebrate trawl bycatch off Cuddalore, Parangipettai and Pazhayar, south-east coast of India. Indian J. Fish. 60 (1), 41-49.

Pillai, N.S., 1998. Bycatch reduction devices in shrimp trawling. Fish. Chimes 18 (7), 45-47.

Pillai, S.L., Kizhakudan, S.J., Radhakrishnan, E.V., Thirumilu, P., 2014. Crustacean bycatch from trawl fishery along north Tamil Nadu coast. Indian J. Fish.. 61 (2), 7-13.

Rajeswari, G., Raghu Prakash, R., Sreedhar, U., 2010. Evaluation of square mesh cod ends for bycatch reduction in demersal trawling off Andhra Pradesh, India. In: Meenakumari, B., Boopendranath, M.R., Edwin, L., Sankar, T.V., Gopal, N., Ninan, G. (Eds.), Coastal Fisheries Resources of India: Conservation and Sustainable Utilisation. Society of Fisheries Technologists, India, Cochin, pp. 341-351.

Ramkumar, S., Ranjith, L., Jaiswar, A.K., Vinod, K., Deshmukh, V., 2019. Does the mechanised trawl target the non-targets from the commercial fishing grounds of northern Maharashtra, eastern Arabian Sea India. J. Ent. Zoo. Std. 7 (6), 1133-1140.

Ramkumar, S., Sundaram, S., Jaiswar, A.K., Ranjith, L., Chakraborty, S.K., Vinod, K., 2015. Evaluation of economic impact on juvenile landings of cephalopods in Mumbai waters, northwest coast of India. Curr. World Environ. 10 (3), 1004-1010.

Ranjith, L., Shukla, S.P., Vinod, K., Ramkumar, S., Chakraborty, S.K., 2018. Targeting the non-target plant biota: Ecological implications of trawl fishery along Thoothukudi, Southeast coast of India. Regul. Stud. Mar. Sci. 24, 143-155.

Rema Devi, K., 1992. Gobioids of Ennore estuary and its vicinity. Rec. Zool. Surv. India 90 (1-4), 161-189.

Salagrama, V., 1998. Bycatch utilisation in Indian fisheries: an overview. In: Report and Proceedings of FAO/DFID Expert Consultation on Bycatch Utilisation in Tropical Fisheries, Beijing, China, pp. 21-23.

Samanta, R., Chakraborty, S.K., Shenoy, L., Nagesh, T.S., Behera, S., Bhoumik, T.S., 2018. Bycatch characterization and relationship between trawl catch and lunar cycle in single day Shrimp Trawls from Mumbai Coast of India. Regul. Stud. Mar. Sci. 17, 47-58.

Sambandamoorthy, P., Nagamuthu, J., Arumugam, S., Perumal, M., 2015. Diversity and trophic level of ichthyofauna associated with the trawl bycatches of Cuddalore and Parangipettai, south-east coast of India. Mar. Bio. Rec. 8 (1-8), e78. http://dx.doi.org/10.1017/S1755267215000159.

Sekharan, K.V., 1962. On the oil sardine fishery of the Calicut area during the years 1955-56 to 1958-59. Indian J. Fish. 9 (2), 679-700.

Sirajudheen, T.K., Biju Kumar, A., 2014. Diversity of marine ornamental fishes associated with trawl by-catch landings in Neendakara fishing harbour, Kerala. J. Aquat. Biol. Fish. 2 (1), 247-255.

Sivasubramaniam, K., 1990. Biological Aspects of Shrimp Trawl By-Catch. Bay of Bengal News (FAO/BOBP.

Stobutzki, I.C., Miller, M.J., Jones, P., Salini, J.P., 2001. Bycatch diversity and variation in a tropical Australian penaeid shrimp fishery: implications of monitoring. Fish. Res. 53, 283-301.

Sujatha, K., 1996. Trash fish catch of the trawl fishery off Visakhapatnam. J. Aquat. Biol. 11 (1-2), 17-23.

Sukumaran, K.K., Telang, K.Y., Thippeswamy, O., 1982. Trawl Fishery of South Kanara with Special Reference to Prawns and Bycatches. In: Mar. Fish. Inf. Serv. Tech. Ext. Ser., vol. 44, pp. 8-14.

Talwar, P.K., Kacker, R.K., 1984. Commercial Sea Fishes of India. Zoological Survey of India, Calcutta, The Director.

Van Denderen, P.D., 2015. Ecosystem Effects of Bottom Trawl Fishing (Doctoral dissertation). Wageningen University.

Varghese, M., Ranjith, L., Joshi, K.K., 2017. Diversity of reef fishes in trap fishery at Keelakarai, Gulf of Mannar, south-east coast of India. Indian J. Fish. 64 (1), 23-30.

Velip, D.T., Rivonker, C.U., 2015. Rends and composition of trawl bycatch and its implications on tropical fishing grounds off Goa, India. Regul. Stud. Mar. Sci. 2, 65-75. 
Violle, C., Navas, M.L., Vile, D., Kazakou, E., Fortunel, C., Hummel, I., Garnier, E., 2007. Let the concept of trait be functional!. Oikos 116, 882-892. http: //dx.doi.org/10.1111/j.2007.0030-1299.15559.x.

Walter, R.P., Haynes, J.M., 2006. Fish and coral community structure are related on shallow water patch reefs near San Salvador. Bahamas. Bull. Mar. Sci. 79, 365-374.
Zacharia, P.U., Krishnakumar, P.K., Durgekar, N.R., Anoop, A.K., Muthiah, C., 2006. Assessment of bycatch and discards associated with bottom trawling along Karnataka coast, India. In: Kurup, B.M, Ravindran, K. (Eds.), Sustain Fish. School of Industrial Fisheries, CUSAT, Kochi, pp. 434-445. 